\title{
Finite quantum theory of fields and strings. 1. Consistent quantization in the Stueckelberg-Feynman treatment
}

\author{
Zahid Zakir ${ }^{2}$
}

\begin{abstract}
In standard quantum field theory, where free quanta have only positive energy, the antiparticle operators were introduced "manually" and this led to the diverging zero-point energy, which meant the inconsistency of the theory. In the Stueckelberg-Feynman (SF) treatment the positive-energy antiparticles are described as the negative energy particles going backward in time and here some Lagrangians do not lead to the zero-point energy. But earlier it was believed that this treatment leads to a negative norm of states and therefore is also inconsistent. In the paper a consecutive method of canonical quantization of fields and strings in the SF treatment is formulated, where a correct choice of the action function makes the norm of states positive and the choice of a minimal Lagrangian makes it free from the zero-point energy. The symmetric chronological product of operators is introduced, turning into ordinary chronological at forward and antichronological at backward in time evolution. This leads to the causal SF propagator and to the standard diagram technique for interacting fields. String theories containing the zero-point energy of modes are inconsistent, since regularization is impossible at Planck distances due to the inevitable presence of gravity. At quantization of strings in SF treatment, there are models without zero-point energy, which are therefore finite and only consistent, but they do not have a conformal anomaly and critical dimension. The effects attributed to the zero-point energy (Lamb shift, Casimir effect) are explained across the contributions of the fields of real sources and confirm the lack of zero-point vacuum energy. This partly solves the cosmological constant problem. From the SF treatment one can turn to the antiparticle picture by means of the charge conjugation or crossing symmetries. The main consequences of the proposed consistent method of quantization of fields and strings are discussed.

Keywords: methods of quantization, quantum field theory, zero-point energy, cosmological constant, antiparticles, composite models, Casimir effect, chronological ordering, propagators, string theory, conformal anomaly, higher dimensions
\end{abstract}

\section{Content}

Introduction

1. Quantization of two signs of energy systems in the SF treatment ......................................4

1.1. The SF treatment of two signs of energy states............................................................ 4

1.2. Oscillators with real-valued coordinates have the zero-point energy ........................... 6

1.3. Oscillators with complex coordinates without zero-point energy................................... 8

2. Quantization of fields in SF treatment without zero-point energy ........................................9

2.1. Real-valued scalar field with zero-point energy .................................................... 9

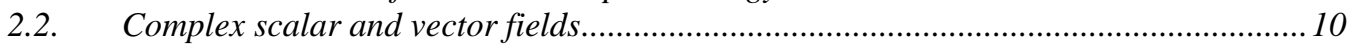

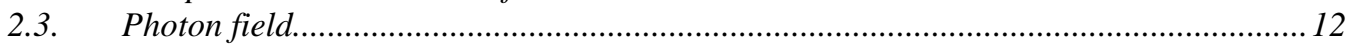

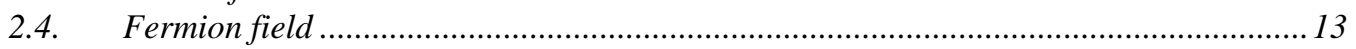

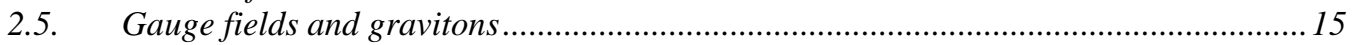

2.6. Scalar fields leading to the spontaneous symmetry breaking ...................................... 15

3. Commutators of fields, propagators, microcausality and statistics ...................................16

3.1. Commutators and causal correlators for the coordinate of oscillator ........................... 16

3.2. The inconsistency of the former standard treatment of microcausality ......................... 18

3.3. The inconsistency of the former proof of the spin and statistics theorem ..................... 19

\footnotetext{
${ }^{1}$ Revised February 8, 2021.

${ }^{2}$ Center for Theoretical Physics and Astrophysics, Tashkent Uzbekistan, zzakir@ qgph.org, ORCID
} 
3.4. Commutators and causal propagators of fields ......................................................20

3.5. The consistency of the treatment of microcausality in the new method ........................20

3.6. Interacting fields and diagram technique ...............................................................21

4. The consistent transition from the SF treatment to the antiparticles ................................21

4.1. Transition to the antiparticles by means of charge conjugation symmetry...................22

4.2. Transition to antiparticles by using crossing symmetry ............................................22

5. On the consequences of the lack of zero-point energy of fields..........................................24

5.1. The experiments confirmed the lack of the zero-point energy of fields ..........................24

5.2. Partial solution of the cosmological constant problem.................................................25

5.3. Supersymmetry does not solve the zero-point energy problem ..................................26

6. Finite quantum theory of strings in the SF treatment ......................................................26

6.1. Inconsistency of the string theories with zero-point energy .........................................26

6.2. Consistent quantization of strings without zero-point energy and anomalies...............27

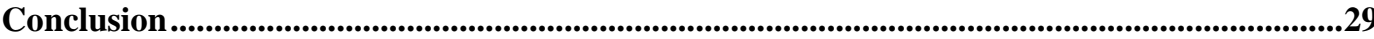

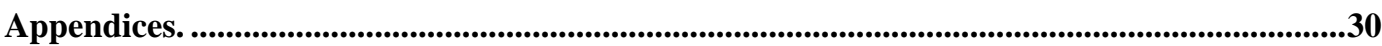

A. Oscillator with two signs of energy in quantum mechanics ..........................................30

B. Zero-point energy as a source of anomalies and critical dimensions of strings ..............31

References

\section{Introduction}

At the first attempts to formulate the relativistic quantum theory, it became clear that the states of both signs of energy enter it on the same basis. For this reason, Dirac proposed the idea of treating the negative energy quanta as some method of describing the positiveenergy antiparticles [1]. This idea was then confirmed at discovering the positron and other antiparticles. The presence of antiparticles made the theory of many-particle one and even a field theory, and, as a result, the quantum theory of relativistic systems became quantum field theory (QFT).

But the negative energy states led to two serious problems, each of which made the theory inconsistent. The first one is the continuous fall of particles into these states. The hypothesis of a filled vacuum and holes in it, as a realization of the Dirac's idea, solved this problem for fermions [1], but crashed in the case of bosons [2]. The second was the problem of negative probabilities, since conventional quantization led to a negative norm for such states [3].

Therefore, in order for QFT to be a consistent physical theory, these problems must be solved. But they seemed unsolvable, and therefore a compromise formulation, operating only with particles and antiparticles of positive energy, was accepted as a standard one. Unfortunately, this led to a new problem with even more catastrophic consequences. It turned out that a plausible ansatz, replacing the operators of negative-energy particles with operators of antiparticles, generates the zero-point energy, even when the formulation with negative energies did not contain it. The standard formulation of QFT, thus, predicted the diverging zero-point energy, making this theory essentially inconsistent.

A formal avoidance of this problem by ignoring the divergent part of the vacuum energy (by the "normal ordering" recipe) does not save the theory, since the inclusion of gravity is inevitable at small distances with high energy fluctuations. Particularly this leads to the cosmological constant problem (more precisely, catastrophe). Numerous attempts to exclude the zero-point energy by means of various hypotheses were unsuccessful, and not only due to the lack of an experimental basis, but mainly due to creating even more problems, as a rule, even more hopeless.

For this reason, there have been many attempts to return to the initial picture with the particles of both signs of energy, somehow solving the old problems with negative energies. Unfortunately, inconsistencies in these attempts led to unrealistic hypotheses for 


\section{Consistent quantization in the Stueckelberg-Feynman treatment}

overcoming their undesirable consequences, which inspired the impression of unrealism for the whole direction of searches.

The character of these inconsistencies was mathematical and/or physical. The main mathematical problem was the negative norm of the negative energy states. The origin of this problem was found and eliminated by Pavšič in 1998 [4]. He started with a two-sign Lagrangian and corresponding Hamiltonian, and showed that in this case the norms of all states remain positive. But in [4], as in many other attempts, there was inconsistency in the physical treatment of the negative energy states.

At the same time, a consistent physical treatment of such states was already discovered in the 1940s. by Stueckelberg and Feynman (SF) [5,6]. In the SF treatment the negative energy particles formally evolve backward in time only and describe the positive energy antiparticles evolving forward in time only. Then the transition of ordinary particles to the negative energy states corresponds to the annihilation of a positive energy particleantiparticle pair.

In the paper it is presented a new method of consistent quantization of systems with states of both signs of energy, in which the generality of the canonical formalism of QFT is joined with the advantages of the SF treatment. At beginning the formalism is similar to [4] and, therefore, all states have a positive norm, but the negative energy states are treated according SF. It is also important that the property of states of different signs of energy to evolve in the opposite directions of time is taken into account starting with the action function. All this partially changes the formalism of the theory and, since some of Lagrangians do not lead to the zero-point energy, finally makes QFT a mathematically correct and physically consistent theory.

One of the changes in formalism is the introduction of a time-symmetric chronological products of operators, which gives the ordinary chronological products in the forward [7] and anti-chronological ones in the backward in time evolution. They lead to the SF causal propagator and the standard diagram technique for interacting fields.

Theories of relativistic strings in former treatments contained the diverging zeropoint energy of ground states, and therefore they were also inconsistent since at small distances regularization is impossible due to the inevitable presence of gravity at Planck distances. Moreover, the zero-point energies led to the anomaly and central charge, and their cancellation condition gave a critical dimension [8]. In the new quantization method, a minimal Lagrangian does not lead to the zero-point energy and the string theory becomes consistent and simple, but it no longer has a conformal anomaly and critical dimension.

It will be shown also that a number of observable effects, such as the Lamb shift and the Casimir effect, attributed to the zero-point fluctuations [9,10], are in fact fully explained by the contributions of the fields of real sources - the loop corrections and the Van der Waals forces. The zero-point fluctuations of the vacuum did not appear in any experiment, since their results would almost double. Thus, their absence, following from the new quantization method, is confirmed by the existing experiments.

All this shows that the new consecutive quantization method leads to a finite and consistent quantum theory of free fields and strings. Two methods of transition from the SF treatment to the antiparticle picture are also presented, the first based on the charge conjugation $(C)$ symmetry and the second using the crossing symmetry.

In Section 1 the new quantization method is describes in the case of harmonic oscillators having two signs of energy states. In Sections 2 and 3, the method is applied to relativistic fields and the causal propagators are constructed. In Section 4 two methods of transition to the picture of antiparticles is presented, and in Section 5 some consequences of the lack of the zero-point energy for quantum fields and strings are discussed. In Section 
6 a consistent string theory without zero-point energy is formulated. In the Appendices the oscillator with two signs of energy in quantum mechanics is described and the zero-point energy as a source of anomalies and critical dimensions in the string theory is discussed.

\section{Quantization of two signs of energy systems in the SF treatment}

\subsection{The SF treatment of two signs of energy states}

The description of evolution in time of a physical system from the initial moment $t_{0}$ to the final moment $t_{1}$ begins with the construction of an action function $S$, defined as the time integral from the Lagrange function $L$ :

$$
S=\int_{t_{0}}^{t_{1}} L d t
$$

In this case, it was usually assumed that evolution goes forward in time only and therefore the final moment is in the future with respect to $t_{0}$, i.e. $t_{1}>t_{0}$. This is how a system of the positive energy particles and antiparticles, having the Lagrangians $L_{+}\left(q_{+}, \dot{q}_{+}\right)$and $L_{a+}\left(q_{a+}, \dot{q}_{a+}\right)$ respectively, is described. Here, $L_{+}$is expressed in terms of generalized coordinates and velocities of the particles $q_{+}, \dot{q}_{+}$, and $L_{a+}$ of variables of the antiparticles $q_{a+}, \dot{q}_{a+}$. Evolution here goes forward in time only, i.e. from the present moment $t_{0}$ to the future moment $t_{1}$ and therefore the direction of integration in the expression for the action function $S$ should be fixed by means a step function $\theta\left(t_{1}-t_{0}\right)$ :

$$
S=\theta\left(t_{1}-t_{0}\right) \int_{t_{0}}^{t_{1}} L_{+} d t+\theta\left(t_{1}-t_{0}\right) \int_{t_{0}}^{t_{1}} L_{a+} d t
$$

In the relativistic theory, however, the introduction of two types of objects, particles and antiparticles, is not necessary, since it is possible to deal with one type of object only, for example, with particles, which is much more convenient. Antiparticles formally behave like the same particles, but with negative energy and going backward in time and this property lies on the basis of the SF treatment $[5,6]$. This treatment appears as a natural and very convenient in particle physics.

The SF treatment considers the evolution of one type of objects in both directions of time and the final moment may be in the past too, i.e. $t_{1}<t_{0}$. In this more general case, the integral in (1) will be the sum of two terms, where in the first integration goes from the present moment $t_{0}$ to the future $t_{1}>t_{0}$, and in the second to the past $t_{1}<t_{0}$.

In contrast to the initial and subsequent formulations of the SF treatment, in this paper the main idea of this treatment will be implemented already starting with the action function, i.e. constructing a correct expression for it as a time integral from the Lagrangian.

To do this, in (2) in the second integral $t_{1}$ we will present as $t_{1}=t_{0}+\Delta t$, where $\Delta t>0$, and we perform the following operations, leaving the action function unchanged:

a) rearrange the limits of integration, changing the sign of the integral;

b) using translational symmetry, shift both limits of the integral down to $\Delta t$ by subtracting $\Delta t$; 
c) introduce the Lagrangian of the negative energy particles $L_{-}\left(q_{-}, \dot{q}_{-}\right)$, equating it to the opposite sign Lagrangian of antiparticles, i.e. $L_{-}\left(q_{-}, \dot{q}_{-}\right)=-L_{a+}\left(q_{a+}, \dot{q}_{a+}\right)$ :

$$
\int_{t_{0}}^{t_{1}} L_{a+} d t=\int_{t_{0}}^{t_{0}+\Delta t} L_{a+} d t=-\int_{t_{0}+\Delta t}^{t_{0}} L_{a+} d t=\int_{t_{0}}^{t_{0}-\Delta t}\left(-L_{a+}\right) d t=\int_{t_{0}}^{t_{0}-\Delta t} L_{-} d t .
$$

d) make similar changes in the step function at the second integral in (2) and redenote $t_{1}=t_{0}-\Delta t$ :

$$
\theta\left(t_{1}-t_{0}\right)=\theta\left(t_{0}+\Delta t-t_{0}\right)=\theta\left[t_{0}-\left(t_{0}-\Delta t\right)\right] \rightarrow \theta\left(t_{0}-t_{1}\right)
$$

As a result, the action function (2) takes the form required by the $S F$ treatment:

$$
S=\theta\left(t_{1}-t_{0}\right) \int_{t_{0}}^{t_{1}} L_{+} d t+\theta\left(t_{0}-t_{1}\right) \int_{t_{0}}^{t_{1}} L_{-} d t
$$

Since $t_{1}<t_{0}$ in the second term of Eq. (5), the time integration essentially goes in the opposite direction, from the present $t_{0}$ to the past $t_{1}$.

Notice, that the choice of particles is conditional, since we could do the opposite choice, expressing everything in terms of antiparticles, by assuming that the particles are the negative energy antiparticles going backward in time, and (2) would turn to:

$$
S=\theta\left(t_{0}-t_{1}\right) \int_{t_{0}}^{t_{1}} L_{a-} d t+\theta\left(t_{1}-t_{0}\right) \int_{t_{0}}^{t_{1}} L_{a+} d t .
$$

The main requirement for the SF treatment is the mutually opposite direction of the time evolution of states with different signs of energy. For a free relativistic particle, having also has an antiparticle, the classical action function takes the form:

$$
S=-\theta\left(t_{1}-t_{0}\right) m c^{2} \int_{t_{0}}^{t_{1}} d t \sqrt{1-v_{+}^{2} / c^{2}}+\theta\left(t_{0}-t_{1}\right) m c^{2} \int_{t_{0}}^{t_{1}} d t \sqrt{1-v_{-}^{2} / c^{2}}
$$

The Hamilton-Jacobi equation for $S=S_{+}+S_{-}$has the form:

$$
\begin{gathered}
\frac{\partial S_{ \pm}}{\partial t}+H_{ \pm}=0, \\
H_{ \pm}= \pm \frac{m c^{2}}{\sqrt{1-\mathbf{v}_{ \pm}^{2} / c^{2}}}= \pm \sqrt{\mathbf{p}_{ \pm}^{2}+m^{2} c^{4}}, \quad \mathbf{p}_{ \pm}=\frac{\partial L_{ \pm}}{\partial \mathbf{v}_{ \pm}}= \pm \frac{m \mathbf{v}_{ \pm}}{\sqrt{1-\mathbf{v}_{ \pm}^{2} / c^{2}}}, \quad \mathbf{v}_{ \pm}=\frac{ \pm d \mathbf{r}_{ \pm}}{ \pm d t} .
\end{gathered}
$$

Notice that, in $\partial_{t} S$ the time derivative $\partial_{t} \theta\left(t-t_{0}\right)$ gives the $\delta$-function $\delta\left(t-t_{0}\right)$, but it is multiplied to the integral disappearing at $t=t_{0}$.

For a correct understanding and application of the SF treatment, the following facts are important, which are usually not clearly explained in the literature.

1) The SF treatment does not mean that the negative energy particles really exist, but on the contrary, that they do not exist in nature, but there are the positive energy antiparticles, which at described in terms of particles are formally represented as the negative energy states. The worldline of a negative energy particle directed backward in time is only a way of describing the worldline of a positive energy antiparticle directed forward in time.

2) If the theory is formulated in terms of particles of both signs of energy with right choice of the time evolution direction according the SF treatment, then the observables of 
this theory are directly connected with experimental data and there is no need to reformulate it in terms of antiparticles. It is enough to know the fact that the initial state of the negativeenergy particle with $-p_{\mu}$ represents the final state of antiparticle with $+p_{\mu}$ and vice versa.

3) The SF treatment lies on the basis of particle physics and, in particular, the crossing symmetry follows from it, sufficiently simplifying the description of the set of interrelating in this sense processes. By this symmetry, the end of the line in the Feynman diagram in the upper light cone, describing a particle, can be transferred to the lower light cone with a change in the sign of the 4-momentum $p_{\mu} \rightarrow-p_{\mu}$, and then this line, formally describing a particle going backward in time, will in fact describe the positive energy antiparticle going forward in time

4) If we still want to turn to the picture of antiparticles in the matrix elements, then for this it is enough to perform carefully the crossing transformation of these matrix elements by making the required Hermitian conjugations of the products of operators with permutation of the initial and final states.

5) The SF treatment concerns both quantum and classical theory, and not only relativistic systems, but nonrelativistic ones too, if they contain antiparticles. The nonrelativistic positron may be described as a nonrelativistic electron of negative energy, going backward in time, by modifying formalisms of mechanics and quantum mechanics.

\subsection{Oscillators with real-valued coordinates have the zero-point energy}

Consider a harmonic oscillator with two types of coordinates corresponding to the states of two signs of energy. In the SF treatment this model describes a system of particle and antiparticle in a harmonic potential.

The action function of such oscillator is given by (5), where the Lagrangians now have the form $(\dot{x}=d x / d t)$ :

$$
L_{+}=\frac{m}{2}\left(\dot{x}_{+}^{2}-\omega^{2} x_{+}^{2}\right), \quad L_{-}=-\frac{m}{2}\left(\dot{x}_{-}^{2}-\omega^{2} x_{-}^{2}\right),
$$

which was studied in [4]. This gives the expressions for canonical momenta:

$$
p_{+}=\frac{\partial L}{\partial \dot{x}_{+}}=m \dot{x}_{+}, \quad p_{-}=\frac{\partial L}{\partial \dot{x}_{-}}=-m \dot{x}_{-} .
$$

Notice that the momentum of a negative energy state contains a minus sign and further this makes the commutators of the ladder operators positive, which means that both the norm of states and the corresponding probabilities will be positive.

Hamiltonians for two modes take the form:

$$
\begin{aligned}
& H_{+}=p_{+} \dot{x}_{+}-L_{+}=\frac{1}{2 m}\left[p_{+}^{2}+(m \omega)^{2} x_{+}^{2}\right], \\
& H_{-}=p_{-} \dot{x}_{-}-L_{-}=-\frac{1}{2 m}\left[p_{-}^{2}+(m \omega)^{2} x_{-}^{2}\right] .
\end{aligned}
$$

Corresponding canonical equations:

$$
\dot{p}_{+}=-\frac{\partial H_{+}}{\partial x_{+}}=-m \omega^{2} x_{+}, \quad \dot{p}_{-}=-\frac{\partial H_{-}}{\partial x_{-}}=m \omega^{2} x_{-},
$$

after substituting (11), give the similar equations of motion for both modes:

$$
\ddot{x}_{+}+\omega^{2} x_{+}=0, \quad \ddot{x}_{-}+\omega^{2} x_{-}=0 .
$$


Their solutions are also similar and differ only by a sign on $\omega t$ :

$$
\begin{array}{ll}
x_{+}=\frac{1}{\sqrt{2 m \omega}}\left(a_{+} e^{-i \omega t}+a_{+}^{*} e^{i \omega t}\right), & x_{-}=\frac{1}{\sqrt{2 m \omega}}\left(a_{-} e^{i \omega t}+a_{-}^{*} e^{-i \omega t}\right), \\
p_{+}=\frac{-i m \omega}{\sqrt{2 m \omega}}\left(a_{+} e^{-i \omega t}-a_{+}^{*} e^{i \omega t}\right), & p_{-}=\frac{-i m \omega}{\sqrt{2 m \omega}}\left(a_{-} e^{i \omega t}-a_{-}^{*} e^{-i \omega t}\right) .
\end{array}
$$

At quantization, the commutation relations:

$$
\left[x_{+}, p_{+}\right]=i, \quad\left[x_{-}, p_{-}\right]=i,
$$

lead to the commutators for ladder operators, nonzero of which are:

$$
\left[a_{+}, a_{+}^{*}\right]=1, \quad\left[a_{-}, a_{-}^{*}\right]=1 .
$$

The ground state average of the commutators (19) gives the norm for the first excited state, which is positive for quanta of both signs of energy:

$$
\left\langle 0\left|\left[a_{ \pm}, a_{ \pm}^{*}\right]\right| 0\right\rangle=\left\langle 0\left|a_{ \pm} a_{ \pm}^{*}\right| 0\right\rangle=\left\langle 1_{ \pm} \mid 1_{ \pm}\right\rangle=1 .
$$

In the previous formulations of the SF treatment, where the Lagrangian $L_{-}$was positive definite, the commutator $\left[a_{-}, a_{-}^{*}\right]=-1$ had a negative sign and this led to the negative norm $\left\langle 1_{-} \mid 1_{-}\right\rangle=-1$, which meant the inconsistency of such a theory. In the developing in this paper formulation, $L_{-}$is negative-definite and, therefore, both the commutator and the norm are positive: $\left[a_{-}, a_{-}^{*}\right]=1,\left\langle 1_{-} \mid 1_{-}\right\rangle=1$.

The ground states for both modes are defined similarly:

$$
a_{+}|0\rangle=0, \quad a_{-}|0\rangle=0 .
$$

This gives similar equations for the ground states $\psi_{0 \pm}$ :

$$
\frac{1}{\sqrt{2 m \omega}}\left(m \omega x_{ \pm}+\frac{\partial}{\partial x_{ \pm}}\right) \psi_{0 \pm}=0,
$$

accordingly, solutions are similar also and both are normalized to unity:

$$
\psi_{0 \pm}=\left(\frac{m \omega}{\pi}\right)^{1 / 4} \exp \left(-\frac{m \omega}{2} x_{ \pm}^{2}\right), \quad \int_{-\infty}^{\infty} \psi_{0 \pm}^{2} d x_{ \pm}=1 .
$$

Hamiltonians (12)-(13) and their eigenvalues then take the form:

$$
\begin{gathered}
H_{+}=\frac{\omega}{2}\left(a_{+}^{*} a_{+}+a_{+} a_{+}^{*}\right)=\omega\left(a_{+}^{*} a_{+}+\frac{1}{2}\right), \quad E_{n+}=\omega\left(n_{+}+\frac{1}{2}\right), \\
H_{-}=-\frac{\omega}{2}\left(a_{-}^{*} a_{-}+a_{-} a_{-}^{*}\right)=-\omega\left(a_{-}^{*} a_{-}+\frac{1}{2}\right), E_{n-}=-\omega\left(n_{-}+\frac{1}{2}\right),
\end{gathered}
$$

where $n_{ \pm}=0,1,2 \ldots$ are the eigenvalues of the number operators $N_{ \pm}=a_{ \pm}^{*} a_{ \pm}$.

If one would returns to the picture of antiparticles with the action function (2), then instead of (25) one would get the Hamiltonian for antiquanta as an analog of (24):

$$
H_{a+}=\frac{\omega}{2}\left(a_{a+}^{*} a_{a+}+a_{a+} a_{a+}^{*}\right)=\omega\left(a_{a+}^{*} a_{a+}+\frac{1}{2}\right), \quad E_{a, n+}=\omega\left(n_{a+}+\frac{1}{2}\right) .
$$


The zero-point energies of the quanta and antiquanta are positive and, by added together, give the total energy of the ground-state two times larger than for the quanta only:

$$
H=H_{+}+H_{a+}=\omega\left(a_{+}^{*} a_{+}+a_{a+}^{*} a_{a+}+1\right), E_{n+, a n+}=\omega\left(n_{+}+n_{a+}+1\right) .
$$

The transition to the SF picture with the negative energy particles does not change the action function, and at substituting (25) into (5) taking into account (13), the zero-point energies of both modes also summing up and double the ground state energy. This occurs because the integrands can be summed only for the same integration limits, i.e. after rearranging the limits and thereby changing the sign of the second integral in (5), which compensates the sign in (25). Thus, the theory is internally self-consistent, since both pictures, with antiquanta or with negative-energy quanta, give the same result that the zeropoint energy of antiquanta doubles the ground state energy.

Thus, the theory of a harmonic oscillator, expanded by including the negative-energy states, is self-consistent and coincides with the SF treatment, where the signs of energy and directions of particle evolution are the same.

\subsection{Oscillators with complex coordinates without zero-point energy}

Let's the harmonic oscillators with two signs on energy states be described by complex coordinates $q_{ \pm}, q_{ \pm}^{*}$. In the action function of such oscillators (5) we choose the Lagrangians of two types of states in the form:

$$
L_{ \pm}= \pm m\left(\dot{q}_{ \pm}^{*} \dot{q}_{ \pm}-\omega^{2} q_{ \pm}^{*} q_{ \pm}\right) .
$$

The canonical momenta and the Hamiltonian then take the form:

$$
\begin{gathered}
p_{ \pm}=\frac{\partial L}{\partial \dot{q}_{ \pm}}= \pm m \dot{q}_{ \pm}^{*}, \quad p_{ \pm}^{*}=\frac{\partial L}{\partial \dot{q}_{ \pm}^{*}}= \pm m \dot{q}_{ \pm}, \\
H_{ \pm}=p_{ \pm} \dot{q}_{ \pm}+\dot{q}_{ \pm}^{*} p_{ \pm}^{*}-L_{ \pm}= \pm \frac{1}{m}\left(p_{ \pm} p_{ \pm}^{*}+m^{2} \omega^{2} q_{ \pm}^{*} q_{ \pm}\right) .
\end{gathered}
$$

The minus signs in momenta $p_{-}, p_{-}^{*}$ make positive both the commutators of the ladder operators and the norms of states. The equations of motion:

$$
\ddot{q}_{ \pm}+\omega^{2} q_{ \pm}=0, \quad \ddot{q}_{ \pm}^{*}+\omega^{2} q_{ \pm}^{*}=0
$$

lead to the frequency decompositions of coordinates:

$$
\begin{array}{ll}
q=q_{+}+q_{-}=\frac{1}{\sqrt{2 m \omega}}\left(a_{+} e^{-i \omega t}+a_{-} e^{i \omega t}\right), & q_{ \pm}=\frac{1}{\sqrt{2 m \omega}} a_{ \pm} e^{\mp i \omega t}, \\
q^{*}=q_{+}^{*}+q_{-}^{*}=\frac{1}{\sqrt{2 m \omega}}\left(a_{+}^{*} e^{i \omega t}+a_{-}^{*} e^{-i \omega t}\right), & q_{ \pm}^{*}=\frac{1}{\sqrt{2 m \omega}} a_{ \pm}^{*} e^{ \pm i \omega t},
\end{array}
$$

Here $q$, in contrast to (16), includes an operator $a_{-}$instead of $a_{+}^{*}$, since otherwise it would not be complex, whereas $a_{+}^{*}$ enters into the expression for $q^{*}$. Momenta have the form:

$$
\begin{array}{ll}
p=p_{+}+p_{-}=\frac{i m \omega}{\sqrt{2 m \omega}}\left(a_{+}^{*} e^{i \omega t}+a_{-}^{*} e^{-i \omega t}\right), & p_{ \pm}=\frac{i m \omega}{\sqrt{2 m \omega}} a_{ \pm}^{*} e^{ \pm i \omega t}, \\
p^{*}=p_{+}^{*}+p_{-}^{*}=\frac{-i m \omega}{\sqrt{2 m \omega}}\left(a_{+} e^{-i \omega t}+a_{-} e^{i \omega t}\right), & p_{ \pm}^{*}=\frac{-i m \omega}{\sqrt{2 m \omega}} a_{ \pm} e^{\mp i \omega t},
\end{array}
$$

i.e. $p=i m \omega q^{*}$ and $p^{*}=-i m \omega q$. The ladder operators are defined as: 
1. Consistent quantization in the Stueckelberg-Feynman treatment

$$
a_{ \pm}=\frac{e^{ \pm i \omega t}}{\sqrt{2 m \omega}}\left(m \omega q_{ \pm}+i p_{ \pm}^{*}\right), \quad a_{ \pm}^{*}=\frac{e^{\mp i \omega t}}{\sqrt{2 m \omega}}\left(m \omega q_{ \pm}^{*}-i p_{ \pm}\right) .
$$

Quantization leads to the commutators:

$$
[q, p]=i, \quad\left[q^{*}, p^{*}\right]=i, \quad\left[a_{ \pm}, a_{ \pm}^{*}\right]=1 .
$$

Operators of different signs of energy commute, since they act in different space of states. These states of both types of oscillators are determined by the action of the corresponding ladder operators on their ground and excited states $\left|n_{ \pm}\right\rangle \mathrm{c} n_{ \pm}=0,1,2, \ldots$ :

$$
a_{ \pm}\left|0_{ \pm}\right\rangle=0, \quad a_{ \pm}\left|n_{ \pm}\right\rangle=\sqrt{n_{ \pm}}\left|n_{ \pm}-1\right\rangle, \quad a_{ \pm}^{*}\left|n_{ \pm}\right\rangle=\sqrt{n_{ \pm}+1}\left|n_{ \pm}+1\right\rangle, \ldots
$$

The Hamiltonians $H_{ \pm}$and the number operators $N_{ \pm}$take the form:

$$
\begin{aligned}
& H=H_{+}+H_{-}=\omega\left(a_{+}^{*} a_{+}-a_{-}^{*} a_{-}\right), \\
& N=N_{+}+N_{-}=a_{+}^{*} a_{+}+a_{-}^{*} a_{-} .
\end{aligned}
$$

As we see, at choosing the minimum Lagrangian (28), the Hamiltonian (37) does not contain the zero-point energy in the ground state.

The transition from the negative energy quanta to the positive energy antiquanta does not change this conclusion. Indeed, such a transition is performed in the action function (5) by a rearranging of the time integral limits compensating the negative sign of the Lagrangian. Notice that the crossing symmetric transformation in the diagram describing this process does not change a numerical value of the energy of quanta:

$$
\left|\left\langle 0_{-}\left|H_{-}\right| 0_{-}\right\rangle\right|=\left\langle 0_{a+}\left|H_{a+}\right| 0_{a+}\right\rangle .
$$

Thus, the theory of the complex harmonic oscillator with the minimal Lagrangian (28), having the negative energy states, is self-consistent and coincides with the SF treatment. It does not have the zero-point energy both in the picture of quanta of both signs on energy, and in the picture of positive energy quanta and antiquanta.

\section{Quantization of fields in SF treatment without zero-point energy}

\subsection{Real-valued scalar field with zero-point energy}

Before proceeding to the complex fields, which may not have the zero-point energy, as in the case of the oscillator with a complex coordinate, we consider a real-valued field, which, like the oscillator with the real-valued coordinate, has the zero-point energy. In particle physics this field can describe only composite particles, while in condensed states - quasiparticles and quasi-antiparticles.

For the real-valued scalar relativistic field $\phi$ the action function in the SF treatment (5) includes the Lagrangians $L_{ \pm}$for two types of field states, similar to the case of a chain of complex oscillators:

$$
L_{ \pm}= \pm \frac{1}{2} \int d^{3} x\left[\left(\partial^{\mu} \phi_{ \pm}\right)^{2}-m^{2} \phi_{ \pm}^{2}\right] .
$$

Corresponding Hamiltonian and generalized momenta of the field are:

$$
H_{ \pm}= \pm \frac{1}{2} \int d^{3} x\left[\pi_{ \pm}^{2}+\left(\nabla \phi_{ \pm}\right)^{2}+m^{2} \phi_{ \pm}^{2}\right]
$$




$$
\pi_{ \pm}(x)=\frac{\partial L}{\partial\left(\partial_{t} \phi_{ \pm}\right)}= \pm \partial_{t} \phi_{ \pm} .
$$

Field equations and simultaneous commutators have the form:

$$
\begin{gathered}
\left(\partial_{\mu} \partial^{\mu}-m^{2}\right) \phi=0, \\
{\left[\phi(\mathbf{x}, t), \pi\left(\mathbf{x}^{\prime}, t\right)\right]=i \delta^{3}\left(\mathbf{x}-\mathbf{x}^{\prime}\right) .}
\end{gathered}
$$

Solutions of the equations lead to frequency decompositions of fields and their momenta:

$$
\begin{gathered}
\phi_{+}=\sum_{k}\left(a_{k} e^{-i k x}+a_{k}^{*} e^{i k x}\right), \quad \phi_{-}=\sum_{k}\left(a_{-k} e^{i k x}+a_{-k}^{*} e^{-i k x}\right), \\
\pi_{+}=-i \sum_{k} \omega_{k}\left(a_{k} e^{-i k x}-a_{k}^{*} e^{i k x}\right), \quad \pi_{-}=-i \sum_{k} \omega_{k}\left(a_{-k} e^{i k x}-a_{-k}^{*} e^{-i k x}\right),
\end{gathered}
$$

where $k=\left(\omega_{\mathbf{k}}, \mathbf{k}\right), \omega_{\mathbf{k}}=\left(\mathbf{k}^{2}+m^{2}\right)^{1 / 2}$, and $\sum_{k}=\int d^{3} k\left[(2 \pi)^{3} 2 \omega_{k}\right]^{-1 / 2}$.

Substituting (44)-(45) into (43) gives the commutators for the creation and annihilation operators of field's quanta of both signs on energy, nonzero of which are:

$$
\left[a_{k}, a_{k^{\prime}}^{*}\right]=\left[a_{-k}, a_{-k^{\prime}}^{*}\right]=\delta^{3}\left(\mathbf{k}-\mathbf{k}^{\prime}\right) .
$$

The energies of field's states (40) are expressed through these operators in the form:

$$
\begin{gathered}
H_{+}=\frac{1}{2} \int d^{3} k \omega_{k}\left(a_{k}^{*} a_{k}+a_{k} a_{k}^{*}\right)=\int d^{3} k \omega_{k}\left(a_{k}^{*} a_{k}+\frac{1}{2}\right), \\
H_{-}=-\frac{1}{2} \int d^{3} k \omega_{k}\left(a_{-k}^{*} a_{-k}+a_{-k} a_{-k}^{*}\right)=-\int d^{3} k \omega_{k}\left(a_{-k}^{*} a_{-k}+\frac{1}{2}\right) .
\end{gathered}
$$

The vacuum states are defined as $a_{k}|0\rangle=0, a_{-k}|0\rangle=0$ and the norm of all states is positive. As for a real-valued harmonic oscillator in Section 1.2, the zero-point vacuum energies of two types of states, particles and antiparticles, are also positive and add up.

\subsection{Complex scalar and vector fields}

In the case of a relativistic scalar field $\phi$, the energy of quanta $p_{0}$, in contrast to the nonrelativistic case, is related with the momentum $\mathbf{k}$ by the relativistic formula and a complete set of solutions of field equations includes states of both signs of energy $p_{0 \pm}= \pm \omega_{\mathbf{k}}$. Particles with $p_{0-}$ in the SF treatment go backward in time and describe antiparticles with $p_{0+}$ going forward in time.

For this reason, in a general case the relativistic fields are described by complex field functions. This is true even for fields with truly neutral particles, since true neutrality in this case means the practical indistinguishability of particles and antiparticles only.

For a complex scalar relativistic field $\phi$ with an action function in the SF treatment (5), the Lagrangians for two types of field states can be chosen as minimal ones:

$$
L_{ \pm}= \pm \int d^{3} x\left(\partial_{\mu} \phi_{ \pm}^{*} \cdot \partial^{\mu} \phi_{ \pm}-m^{2} \phi_{ \pm}^{*} \phi_{ \pm}\right) .
$$

The corresponding Hamiltonian, charge operator, and momenta are equal to: 


$$
\begin{gathered}
H_{ \pm}= \pm \int d^{3} x\left(\pi_{ \pm} \pi_{ \pm}^{*}+\nabla \phi_{ \pm}^{*} \cdot \nabla \phi_{ \pm}+m^{2} \phi_{ \pm}^{*} \phi_{ \pm}\right), \\
Q_{ \pm}= \pm i \int d^{3} x\left(\phi_{ \pm}^{*} \pi_{ \pm}^{*}-\pi_{ \pm} \phi_{ \pm}\right), \\
\pi_{ \pm}(x)=\frac{\partial L}{\partial\left(\partial_{t} \phi_{ \pm}\right)}= \pm \partial_{t} \phi_{ \pm}^{*}, \pi_{ \pm}^{*}(x)=\frac{\partial L}{\partial\left(\partial_{t} \phi_{ \pm}^{*}\right)}= \pm \partial_{t} \phi_{ \pm} .
\end{gathered}
$$

Field equations and simultaneous field commutators have the form:

$$
\begin{gathered}
\left(\partial_{\mu} \partial^{\mu}-m^{2}\right) \phi=0, \quad\left(\partial_{\mu} \partial^{\mu}-m^{2}\right) \phi^{*}=0 . \\
{\left[\phi(\mathbf{x}, t), \pi\left(\mathbf{x}^{\prime}, t\right)\right]=\left[\phi^{*}(\mathbf{x}, t), \pi^{*}\left(\mathbf{x}^{\prime}, t\right)\right]=i \delta^{3}\left(\mathbf{x}-\mathbf{x}^{\prime}\right) .}
\end{gathered}
$$

Solutions of field equations lead to the frequency decomposition of the field functions:

$$
\begin{array}{ll}
\phi(x)=\phi_{+}+\phi_{-}=\sum_{k}\left(a_{k} e^{-i k x}+a_{-k} e^{i k x}\right), & \phi_{ \pm}=\sum_{k} a_{ \pm k} e^{\mp i k x}, \\
\phi^{*}(x)=\phi_{+}^{*}+\phi_{-}^{*}=\sum_{k}\left(a_{k}^{*} e^{i k x}+a_{-k}^{*} e^{-i k x}\right), & \phi_{ \pm}^{*}=\sum_{k} a_{ \pm}^{*} e^{ \pm i k x},
\end{array}
$$

and the canonical momenta have the form:

$$
\begin{array}{ll}
\pi(x)=\pi_{+}+\pi_{-}=i \sum_{k} \omega_{k}\left(a_{k}^{*} e^{i k x}+a_{-k}^{*} e^{-i k x}\right), & \pi_{ \pm}=i \sum_{k} \omega_{k} a_{ \pm}^{*} e^{ \pm i k x}, \\
\pi^{*}(x)=\pi_{+}^{*}+\pi_{-}^{*}=-i \sum_{k} \omega_{k}\left(a_{k} e^{-i k x}+a_{-k} e^{i k x}\right), & \pi_{ \pm}^{*}=-i \sum_{k} \omega_{k} a_{ \pm k} e^{\mp i k x},
\end{array}
$$

Substituting (54)-(55) into (53), we obtain the commutators for the creation and annihilation operators of field quanta of both signs on energy, nonzero of which are:

$$
\left[a_{k}, a_{k^{\prime}}^{*}\right]=\left[a_{-k}, a_{-k^{\prime}}^{*}\right]=\delta^{3}\left(\mathbf{k}-\mathbf{k}^{\prime}\right) .
$$

The energy and charge (50) are expressed through these operators in the form:

$$
\begin{aligned}
& H=H_{+}+H_{-}=\int d^{3} k\left(a_{k}^{*} a_{k}-a_{-k}^{*} a_{-k}\right) \omega_{k}, \\
& Q=Q_{+}+Q_{-}=\int d^{3} k\left(a_{k}^{*} a_{k}+a_{-k}^{*} a_{-k}\right) .
\end{aligned}
$$

The annihilation operators define the vacuum for both types of particles:

$$
a_{k}|0\rangle=0, \quad a_{-k}|0\rangle=0,
$$

which, unlike the real-valued scalar field, do not contain a zero-point energy and a zeropoint charge due to the chosen minimal form of the Lagrangians (49).

Thus, the operators of observables of the complex scalar field in the SF treatment are normal ordered, and the energy and charge of the vacuum vanish.

The complex vector field is expanded in normal modes similar to the complex scalar field, taking into account the polarization vector $\varepsilon_{\mu k}^{\lambda}$ :

$$
\begin{aligned}
& B_{\mu}(x)=\sum_{\mathbf{k} \lambda}\left(a_{k \lambda} \varepsilon_{\mu k}^{\lambda} e^{-i k x}+a_{-k \lambda} \varepsilon_{\mu k}^{\lambda^{*}} e^{i k x}\right), \\
& B_{\mu}^{*}(x)=\sum_{\mathbf{k} \lambda}\left(a_{k \lambda}^{*} \varepsilon_{\mu k}^{\lambda^{*}} e^{i k x}+a_{-k \lambda}^{*} \varepsilon_{\mu k}^{\lambda} e^{-i k x}\right) .
\end{aligned}
$$

After separating the independent degrees of freedom, the equations for the free field are linear for three polarizations and are quantized independently in the given inertial frame as 
three complex scalar fields. Therefore, the Hamiltonian and charge operator of the vector field have the form:

$$
\begin{aligned}
& H=\sum_{\lambda} \int d^{3} k\left(a_{k \lambda}^{*} a_{k \lambda}-a_{-k \lambda}^{*} a_{-k \lambda}\right) \omega_{k}, \\
& Q=\sum_{\lambda} \int d^{3} k\left(a_{k \lambda}^{*} a_{k \lambda}+a_{-k \lambda}^{*} a_{-k \lambda}\right),
\end{aligned}
$$

which also do not contain a zero-point energy and zero-point charge.

Although this result was obtained in one a reference frame, the lack of the zero-point vacuum energy and charge does not depend on the choice of reference frame.

\subsection{Photon field}

Let's consider the action function (5) of the SF treatment for electromagnetic field. The Lagrangians for the fields $A_{ \pm}^{\mu}$ of both signs on energy $\left(k^{0}= \pm \omega_{\mathbf{k}}\right)$ have the form:

$$
L_{ \pm}=\mp \frac{1}{4} \int d^{3} x F_{\mu \nu}^{ \pm} F_{ \pm}^{\mu v}= \pm \frac{1}{2} \int d^{3} x \partial_{\mu} A_{ \pm}^{v} \cdot \partial^{\mu} A_{v \pm},
$$

where $F_{ \pm}^{\mu v}=\partial^{\mu} A_{ \pm}^{v}-\partial^{v} A_{ \pm}^{\mu}$ and the standard condition $\partial_{\mu} A_{ \pm}^{\mu}=0$ is used.

In a plane wave the helicity $\Lambda^{0}$, the time component of the divergence of the total angular momentum $\Lambda^{\mu}=\partial_{v} J^{\mu \nu}$, is the projection of the spin $\mathbf{S}$ onto the direction of the momentum: $\Lambda^{0}=\mathbf{S} \cdot \mathbf{k} /|\mathbf{k}|$. At quantization, the circularly polarized waves with two projections of helicity form the pure states of the photon field, in which the Hamiltonian and $\Lambda^{0}$ are diagonal.

Let in the rest frame of photon's source, the spatial axis $x^{3}$ is directed along the photon's momentum $\mathbf{k}=\left(0,0, k^{3}\right)$ and let the gauge $A_{3}=0$ is taken, leaving the transverse physical components $A_{1}, A_{2}$ only. At introducing the complex field $A$ :

$$
A(x)=\left(A_{1}+i A_{2}\right) / \sqrt{2}, \quad A^{*}(x)=\left(A_{1}-i A_{2}\right) / \sqrt{2} .
$$

the photon field Lagrangian (61) takes the form exactly as for a complex scalar field and we again take it in the "minimal" form:

$$
L_{ \pm}= \pm \int d^{3} x \partial_{\mu} A_{ \pm}^{*} \cdot \partial^{\mu} A_{ \pm} \cdot
$$

The free Hamiltonian and the helicity operator then take the form:

$$
\begin{aligned}
& H_{ \pm}= \pm \int d^{3} x\left(\pi_{ \pm} \pi_{ \pm}^{*}+\nabla A_{ \pm}^{*} \cdot \nabla A_{ \pm}\right), \\
& \Lambda_{ \pm}^{0}= \pm i \int d^{3} x\left(A_{ \pm}^{*} \pi_{ \pm}^{*}-\pi_{ \pm} A_{ \pm}\right),
\end{aligned}
$$

where $\pi_{ \pm}(x)= \pm \partial_{t} A_{ \pm}^{*}, \pi_{ \pm}^{*}(x)= \pm \partial_{t} A_{ \pm}$. Photons with opposite helicity $\Lambda^{0}= \pm 1$ behave like states with opposite signs of a chiral charge, and the symmetry between photons of opposite helicities is an analog of the charge conjugation $(C)$ symmetry.

Field equations and the simultaneous field commutators are:

$$
\begin{gathered}
\partial_{\mu} \partial^{\mu} A=0, \quad \partial_{\mu} \partial^{\mu} A^{*}=0, \\
{\left[A(\mathbf{x}, t), \pi\left(\mathbf{x}^{\prime}, t\right)\right]=\left[A^{*}(\mathbf{x}, t), \pi^{*}\left(\mathbf{x}^{\prime}, t\right)\right]=i \delta^{3}\left(\mathbf{x}-\mathbf{x}^{\prime}\right) .}
\end{gathered}
$$


The decomposition of the field into the normal modes gives:

$$
\begin{gathered}
A(x)=\sum_{k}\left(a_{k} e^{-i k x}+a_{-k} e^{i k x}\right), \quad A^{*}(x)=\sum_{k}\left(a_{k}^{*} e^{i k x}+a_{-k}^{*} e^{-i k x}\right), \\
\pi(x)=i \sum_{k} \omega_{k}\left(a_{k}^{*} e^{i k x}+a_{-k} e^{-i k x}\right), \quad \pi^{*}(x)=-i \sum_{k} \omega_{k}\left(a_{k} e^{-i k x}+a_{-k}^{*} e^{i k x}\right) .
\end{gathered}
$$

Here $a_{ \pm k}, a_{ \pm k}^{*}$ are the creation-annihilation operators of photons of the positive helicity $\Lambda_{+}^{0}=+1$, the commutators for which follow from (66) and nonzero of which are:

$$
\left[a_{k}, a_{k^{\prime}}^{*}\right]=\left[a_{-k}, a_{-k^{\prime}}^{*}\right]=\delta^{3}\left(\mathbf{k}-\mathbf{k}^{\prime}\right) .
$$

The Hamiltonian and helicity (64), at inserting (67)-(68), take the form:

$$
\begin{aligned}
& H=H_{+}+H_{-}=\int d^{3} k\left(a_{k}^{*} a_{k}-a_{-k}^{*} a_{-k}\right) \omega_{k}, \\
& \Lambda_{ \pm}^{0}=\Lambda_{+}^{0}+\Lambda_{-}^{0}=\int d^{3} k\left(a_{k}^{*} a_{k}+a_{-k}^{*} a_{-k}\right) .
\end{aligned}
$$

The annihilation operators determine the vacuum: $a_{k}|0\rangle=0, a_{-k}|0\rangle=0$, which does not contain the zero-point energy, the helicity of the vacuum is also vanishes. Due to the invariance of the vacuum properties, the fact of vanishing of the vacuum energy in one a reference frame and in one a gauge, is valid for all reference frames and all gauges.

The described quantization procedure for the photon field in the source's rest frame can be generalized to arbitrary inertial frames and may be presented in the Lorentz covariant form, preserving the axial symmetry of the system, by introducing the tetrads:

$$
\begin{gathered}
A_{1}=e_{1}^{\mu} A_{\mu}, \quad A_{2}=e_{2}^{\mu} A_{\mu}, \quad e_{0}^{\mu} A_{\mu}=e_{3}^{\mu} A_{\mu}=0, \\
A(x)=\frac{1}{\sqrt{2}}\left(e_{1}^{\mu}+i e_{2}^{\mu}\right) A_{\mu}=e_{+}^{\mu} A_{\mu}, \quad A^{*}(x)=\frac{1}{\sqrt{2}}\left(e_{1}^{\mu}-i e_{2}^{\mu}\right) A_{\mu}=e_{-}^{\mu} A_{\mu} .
\end{gathered}
$$

Thus, the photon field, which has two transverse physical components $\left(0, A_{1}, A_{2}, 0\right)$ in the source's rest frame, after the Lorentz transformation to other frames, in a general case has all four components $A_{\mu}=e_{\mu}^{1} A_{1}+e_{\mu}^{2} A_{2}$. The longitudinal component appears due to a different orientation of the spatial coordinate axes, and the time component - due to the Lorentz transformations of the projections $\left(A_{1}, A_{2}\right)$ on the direction of motion of the new inertial frame. Photon propagators will include covariant expressions:

$$
e_{\mu}^{-} e_{v}^{+} A\left(\Lambda x^{\prime}\right) A^{*}(\Lambda x)=e_{\mu}^{a} e_{v}^{b} A_{a}\left(\Lambda x^{\prime}\right) A_{b}(\Lambda x)=A_{\mu}\left(x^{\prime}\right) A_{v}(x) .
$$

Since the tetrad vectors $\left(e_{\mu}^{1}, e_{\mu}^{2}\right)$ are constant kinematic factors, they only introduce kinematic corrections to the quantization procedure and do not change physical results, in particular, do not generate a zero-point energy.

\subsection{Fermion field}

In relativistic theory, the fermion field of spin $1 / 2$ is described by the Dirac spinors $\psi^{+}, \psi$ and in the covariant formulation has two energy signs states. In the action function (5), the Lagrangians of these states have the form:

$$
L=\int d^{3} x\left(\frac{i}{2}\left[\bar{\psi} \gamma^{\mu}\left(\partial_{\mu} \psi\right)-\left(\partial_{\mu} \bar{\psi}\right) \gamma^{\mu} \psi\right]-m \bar{\psi} \psi\right) .
$$


In contrast to the boson case, there is no need to insert the minus sign into $L_{-}$, since the appearance of the negative eigenvalues of the Hamiltonian in the future is contained initially in the construction of spinors $\bar{\psi}_{-}, \psi_{-}$and matrices $\gamma^{\mu}$.

The role of the canonical momentum is played by the conjugated spinor:

$$
\pi=\frac{\partial L}{\partial\left(\partial_{t} \psi\right)}=i \psi^{+}
$$

The Hamiltonian and the charge operator take the form:

$$
H=\frac{i}{2} \int d^{3} x\left[\psi^{+}\left(\partial_{t} \psi\right)-\left(\partial_{t} \psi^{+}\right) \psi\right], \quad Q=\int d^{3} x \psi^{+} \psi .
$$

The corresponding Dirac equations and their solutions are:

$$
\begin{gathered}
\gamma^{\mu} i \partial_{\mu} \psi-m \psi=0, \\
\psi(x)=\psi_{+}+\psi_{-}=m \sqrt{2} \sum_{\alpha p}\left(b_{p \alpha} u_{p}^{\alpha} e^{-i p x}+b_{-p \alpha} v_{p}^{\alpha} e^{i p x}\right), \\
\psi^{+}(x)=\psi_{+}^{+}+\psi_{-}^{+}=m \sqrt{2} \sum_{\alpha p}\left(b_{p \alpha}^{+} u_{p}^{\alpha+} e^{i p x}+b_{-p \alpha}^{+} v_{p}^{\alpha+} e^{-i p x}\right),
\end{gathered}
$$

where the normalization is: $u_{p}^{\alpha+} u_{p}^{\alpha^{\prime}}=v_{p}^{\alpha+} v_{p}^{\alpha^{\prime}}=\delta^{\alpha \alpha^{\prime}} E_{p} / m$ and $E_{p}=\left(\mathbf{p}^{2}+m^{2}\right)^{1 / 2}$. Here, a positive energy state is described by the spinor $u_{p}$, and a negative energy one by $v_{p}$.

In order for quanta to be fermions, i.e. satisfy the Fermi-Dirac statistics, the corresponding creation-annihilation operators, and hence the field operators, must be anticommuting. As a result, when the field operators in the commutators are rearranged, their product changes the sign and the commutator turns to the anti-commutator. Therefore, the field operators are quantized through the simultaneous anticommutators:

$$
\left\{\psi^{\alpha}(\mathbf{x}, t), \pi^{\alpha^{\prime}}\left(\mathbf{x}^{\prime}, t\right)\right\}=i \delta^{3}\left(\mathbf{x}-\mathbf{x}^{\prime}\right) \delta^{\alpha \alpha^{\prime}},
$$

which, taking into account (75), gives:

$$
\left\{\psi^{\alpha}(\mathbf{x}, t), \psi^{+\alpha^{\prime}}\left(\mathbf{x}^{\prime}, t\right)\right\}=\delta^{3}\left(\mathbf{x}-\mathbf{x}^{\prime}\right) \delta^{\alpha \alpha^{\prime}} .
$$

Substitution (78) gives the anticommutators for the creation-annihilation operators:

$$
\left\{b_{ \pm p \alpha}, b_{ \pm p^{\prime} \alpha^{\prime}}^{+}\right\}=\delta^{3}\left(\mathbf{k}-\mathbf{k}^{\prime}\right) \delta_{\alpha \alpha^{\prime}}, \quad\left\{b_{ \pm p \alpha}, b_{ \pm p^{\prime} \alpha^{\prime}}\right\}=\left\{b_{ \pm p \alpha}^{+}, b_{ \pm p^{\prime} \alpha^{\prime}}^{+}\right\}=0
$$

As a result, the Hamiltonian and the charge operator from (76) take the form:

$$
\begin{gathered}
H=\sum_{\alpha} \int d^{3} p\left(b_{p \alpha}^{+} b_{p \alpha}-b_{-p \alpha}^{+} b_{-p \alpha}\right) E_{p}, \\
Q=\sum_{\alpha} \int d^{3} p\left(b_{p \alpha}^{+} b_{p \alpha}+b_{-p \alpha}^{+} b_{-p \alpha}\right) .
\end{gathered}
$$

In this case, the mean vacuum values of the charge and the current operator vanish:

$$
\langle 0|Q(x)| 0\rangle=0, \quad\langle 0|\mathbf{j}(x)| 0\rangle=0 .
$$

Thus, at quantization of the fermion field with the Lagrangians (74), the observables are normal ordered, and there is no zero-point energy and zero-point charge. 


\subsection{Gauge fields and gravitons}

As shown in the Section 2.3, quantization of the electromagnetic field in the new method led to a theory without zero-point vacuum energy. The non-Abelian vector gauge fields $A_{\mu}^{a}$ (spin 1) are similar to the photon field in the free field case and the same result can be expected for them too. The quantization of such gauge fields and the graviton field (spin 2) in the weak-field approximation reduces to quantization of two transverse physical states of vector or tensor fields. All these fields, as massless, have axial symmetry, and at circular polarization the free Hamiltonian and helicity are diagonal.

Therefore, the above described quantization of the photon field is applicable in this case too, and taking into account the nonlinearity and internal symmetries of the fields does not change the main conclusion about the vanishing of vacuum energy. Indeed, nonlinear contributions are proportional to the coupling constant $g$ both in terms of self-interaction in the field strength and in covariant derivatives. Therefore, if we consider weak fields with a coupling constant $g^{2} \ll 1$, then these terms can be neglected and the non-Abelian gauge fields can be quantized as sets of independent photon-like fields.

In the case of gravitons, the effective coupling constant is weak up to the Planch length $l_{g}$, where the gravitational radius of particles is compared with their wavelength, which leads to a strong redshift of the frequencies. A rapid decreasing of the frequencies at distances of order $l_{g}$ due to gravitational freezing of the proper times for external observers, leads to the decreasing the effects of nonlinearity (see the second paper [11]).

Since the vanishing of the vacuum energy of free fields follows from the presence of states of two signs on energy of the relativistic fields, the interaction of quanta does not change this result. All contributions to the vacuum energy density from the interactions will be proportional to the coupling constants and disappear in the weak field limit, while the vacuum energy of the free field does not depend on this factor.

The conclusion that at quantization of the non-Abelian fields and the graviton field in one a reference frame, in the transverse gauge and in the weak-coupling approximation, the zero-point vacuum energy does not arise, remains valid for all reference frames and for all gauges due to the gauge and Lorentz invariance of the vacuum.

\subsection{Scalar fields leading to the spontaneous symmetry breaking}

In the case of systems with vacuum condensates, as well as with strong nonperturbative effects and topologically non-trivial solutions, the contributions to the vacuum energy of the fields can have significant deviations, and they will be discussed further. Nevertheless, the above conclusions from the theory of free fields generally are retained even with these changes. In particular, those contributions that change the vacuum energy to a finite value are not related to the zero-point energy, which diverges by definition.

One such example is the change in the vacuum energy at spontaneous symmetry breaking in the Standard Model. Here, the self-interaction of the doublet of the complex scalar fields leads to the formation of a vacuum condensate, due to the interaction with which masses of particles appear, including some quanta of gauge fields.

However, from the initial doublet, in addition to this condensate, one scalar field remains only, the scalar boson of which is even detected. Such a field in the general case should have the zero-point energy, however, as will be shown below, in this particular case this is not so and it does not have the zero-point energy.

Let's consider spontaneous symmetry breaking in a system with one complex scalar field and an abelian gauge field: 


$$
L_{ \pm}= \pm \int d^{3} x\left(D_{\mu \pm} \phi_{ \pm}^{*} \cdot D^{\mu \pm} \phi_{ \pm}-\frac{\lambda^{2}}{4}\left(\phi_{ \pm}^{*} \phi_{ \pm}-\eta_{ \pm}^{2}\right)^{2}-\frac{1}{4} F_{\mu \nu \pm} F^{\mu \nu \pm}\right) \text {, }
$$

where $D_{\mu \pm}=\partial_{\mu \pm}-i e A_{\mu \pm}, F_{\mu \nu \pm}=\partial_{\mu \pm} A_{\nu \pm}-\partial_{\nu \pm} A_{\mu \pm}$. Writing the complex scalars as:

$$
\phi_{ \pm}(x)=\tilde{\phi}_{ \pm}(x) e^{i \theta_{ \pm}(x)}, \quad \phi_{ \pm}^{*}(x)=\tilde{\phi}_{ \pm}^{*} e^{-i \theta_{ \pm}(x)},
$$

we choose $\theta_{ \pm}{ }^{\prime}$ so that $\tilde{\phi}_{ \pm}^{\prime}$ are real, i.e. $\tilde{\phi}_{ \pm}=\tilde{\phi}_{ \pm}^{*}$ (strokes omitted):

$$
\phi_{ \pm}(x)=\tilde{\phi}_{ \pm}(x) e^{i \theta_{ \pm}(x)}, \quad \phi_{ \pm}^{*}(x)=\tilde{\phi}_{ \pm}(x) e^{-i \theta_{ \pm}(x)} .
$$

We exclude the phase $\theta_{ \pm}$from (85) by a gauge transformation $A_{\mu \pm}=A_{\mu \pm}^{\prime}+e^{-1} \partial_{\mu} \theta_{ \pm}$, and separate from the scalar field $\tilde{\phi}_{ \pm}$a small deviation from $\eta$ :

$$
\tilde{\phi}_{ \pm}(x)=\frac{1}{\sqrt{2}}\left[\eta_{ \pm}+\chi_{ \pm}(x)\right]
$$

Despite $\chi_{ \pm}$being a real-valued scalar field, it is not truly neutral, since it retains a trace about that it was one of the two components of a complex field. Explicitly, its frequency decomposition is given not as for a truly neutral field $\chi \sim \chi_{+} f+\chi_{+}^{*} f^{*}$, but as in the case of a charged field $\chi \sim \chi_{+} f+\chi_{-} f^{*}$. Therefore, such a scalar remainder of the complex field also does not contain a zero-point energy.

Notice, that the particles and antiparticles of such a scalar field in ordinary interactions are indistinguishable, but at $C P$ violation they can lead to observable effects.

\section{Commutators of fields, propagators, microcausality and statistics}

\subsection{Commutators and causal correlators for the coordinate of oscillator}

The commutators for the coordinates of the complex oscillator follow from (32):

$$
\begin{gathered}
{\left[q_{ \pm}\left(t^{\prime}\right), q_{ \pm}^{*}(t)\right]=\frac{1}{2 m \omega}\left[a_{ \pm}, a_{ \pm}^{*}\right] e^{\mp i \omega\left(t^{\prime}-t\right)}=\frac{1}{2 m \omega} e^{\mp i \omega\left(t^{\prime}-t\right)},} \\
{\left[q_{ \pm}\left(t^{\prime}\right), q_{ \pm}(t)\right]=\left[q_{ \pm}^{*}\left(t^{\prime}\right), q_{ \pm}^{*}(t)\right]=0 .}
\end{gathered}
$$

The commutator (89) in the general case $t^{\prime} \neq t$ is complex and becomes real-valued only for equal times. Moreover, complex conjugate coordinates do not commute even at the equal times $\left[q_{ \pm}(t), q_{ \pm}^{*}(t)\right]=1 / 2 m \omega$. As a result of this fact, the order of complex conjugate operators in their products is essential and $q_{ \pm} q_{ \pm}^{*} \neq q_{ \pm}^{*} q_{ \pm}$. The sum of two type coordinates $q=q_{+}+q_{-}$and $q^{*}=q_{+}^{*}+q_{-}^{*}$ also does not commute:

$$
\left[q\left(t^{\prime}\right), q^{*}(t)\right]=\frac{1}{2 m \omega}\left(\left[a_{+}, a_{+}^{*}\right] e^{-i \omega\left(t^{\prime}-t\right)}+\left[a_{-}, a_{-}^{*}\right] e^{i \omega\left(t^{\prime}-t\right)}\right)=\frac{1}{m \omega} \cos \left[\omega\left(t^{\prime}-t\right)\right] .
$$

The causal correlator of coordinates for two moments of time should describe the fact that the positive energy quanta evolve only forward, and the negative energy ones only backward in time. Such a correlator can be constructed if the usual chronological product of operators is generalized to the systems with quanta evolving in different directions of time. 
The usual operator of the chronological product of operators [7], which we denote as $T_{+}$, acts only on the operators of positive energy states. It is not symmetrical in time and arranges the operators in increasing order of the time argument from right to left:

$$
T_{+}\left[A_{+}\left(t_{2}\right) B_{+}\left(t_{1}\right)\right]= \begin{cases}A_{+}\left(t_{2}\right) B_{+}\left(t_{1}\right), & t_{2}>t_{1}, \\ B_{+}\left(t_{1}\right) A_{+}\left(t_{2}\right), & t_{2}<t_{1} .\end{cases}
$$

The operator $T_{-}$, inverse to $T_{+}$, acting only on the negative energy states, we define as the operator ordering the operators in the opposite case - it arranges the operators in decreasing order of the time argument from right to left:

$$
T_{-}\left[A_{-}\left(t_{2}\right) B_{-}\left(t_{1}\right)\right]= \begin{cases}B_{-}\left(t_{1}\right) A_{-}\left(t_{2}\right), & t_{2}>t_{1}, \\ A_{-}\left(t_{2}\right) B_{-}\left(t_{1}\right), & t_{2}<t_{1} .\end{cases}
$$

Their generalization, the symmetric operator of chronological ordering $\hat{T}$, we define as a product of these two operators $T_{+}$and $T_{-}$, which will have, for this reason, the following properties:

$$
\hat{T}=T_{+} T_{-}, \hat{T} A_{+}=T_{+} A_{+}, \hat{T} A_{-}=T_{-} A_{-} .
$$

It acts selectively on the states of both signs of energy, ordering them in a mutually inverse order, for example, at $t_{2}>t_{1}$ :

$$
\begin{aligned}
& \hat{T}\left[A_{+}\left(t_{2}\right) B_{+}\left(t_{1}\right) A_{-}\left(t_{2}\right) B_{-}\left(t_{1}\right)\right]=T_{+}\left[A_{+}\left(t_{2}\right) B_{+}\left(t_{1}\right)\right] \cdot T_{-}\left[A_{-}\left(t_{2}\right) B_{-}\left(t_{1}\right)\right]= \\
& =A_{+}\left(t_{2}\right) B_{+}\left(t_{1}\right) \cdot B_{-}\left(t_{1}\right) A_{-}\left(t_{2}\right)
\end{aligned}
$$

Under the symmetrical chronological product, the operators can be reordered arbitrarily and this does not change the final result.

Causal correlators for the coordinates of a complex oscillator are defined as the matrix elements over the ground state from the symmetrical chronological products at two moments of time $\left\langle 0\left|\hat{T}\left[q\left(t_{2}\right) q^{*}\left(t_{1}\right)\right]\right| 0\right\rangle$ and $\left\langle 0\left|\hat{T}\left[q^{*}\left(t_{2}\right) q\left(t_{1}\right)\right]\right| 0\right\rangle$. At $t_{2}>t_{1}$ they are:

$$
\begin{aligned}
& \left\langle 0\left|\hat{T}\left[q\left(t_{2}\right) q^{*}\left(t_{1}\right)\right]\right| 0\right\rangle=\left\langle 0\left|T_{+}\left[q_{+}\left(t_{2}\right) q_{+}^{*}\left(t_{1}\right)\right]\right| 0\right\rangle+\left\langle 0\left|T_{-}\left[q_{-}\left(t_{2}\right) q_{-}^{*}\left(t_{1}\right)\right]\right| 0\right\rangle= \\
& =\left\langle 0\left|q_{+}\left(t_{2}\right) q_{+}^{*}\left(t_{1}\right)\right| 0\right\rangle+\left\langle 0\left|q_{-}^{*}\left(t_{1}\right) q_{-}\left(t_{2}\right)\right| 0\right\rangle=\left\langle 0\left|q_{+}\left(t_{2}\right) q_{+}^{*}\left(t_{1}\right)\right| 0\right\rangle, \\
& \left\langle 0\left|\hat{T}\left[q^{*}\left(t_{2}\right) q\left(t_{1}\right)\right]\right| 0\right\rangle=\left\langle 0\left|T_{+}\left[q_{+}^{*}\left(t_{2}\right) q_{+}\left(t_{1}\right)\right]\right| 0\right\rangle+\left\langle 0\left|T_{-}\left[q_{-}^{*}\left(t_{2}\right) q_{-}\left(t_{1}\right)\right]\right| 0\right\rangle= \\
& =\left\langle 0\left|q_{+}^{*}\left(t_{2}\right) q_{+}\left(t_{1}\right)\right| 0\right\rangle+\left\langle 0\left|q_{-}\left(t_{1}\right) q_{-}^{*}\left(t_{2}\right)\right| 0\right\rangle=\left\langle 0\left|q_{-}\left(t_{1}\right) q_{-}^{*}\left(t_{2}\right)\right| 0\right\rangle .
\end{aligned}
$$

In the general case, we have:

$$
\left\langle 0\left|\hat{T}\left[q\left(t_{2}\right) q^{*}\left(t_{1}\right)\right]\right| 0\right\rangle=\left\langle 0\left|\hat{T}\left[q^{*}\left(t_{1}\right) q\left(t_{2}\right)\right]\right| 0\right\rangle= \begin{cases}\left\langle 0\left|q_{+}\left(t_{2}\right) q_{+}^{*}\left(t_{1}\right)\right| 0\right\rangle, & t_{2}>t_{1}, \\ \left\langle 0\left|q_{-}\left(t_{2}\right) q_{-}^{*}\left(t_{1}\right)\right| 0\right\rangle, & t_{2}<t_{1} .\end{cases}
$$

Thus, the causal correlator is determined through the symmetrical operator $\hat{T}$ as:

$$
\begin{aligned}
& \left\langle 0\left|\hat{T}\left[q\left(t_{2}\right) q^{*}\left(t_{1}\right)\right]\right| 0\right\rangle=\left\langle 0\left|\hat{T}\left[q^{*}\left(t_{1}\right) q\left(t_{2}\right)\right]\right| 0\right\rangle= \\
& =\theta\left(t_{2}-t_{1}\right)\left\langle 0\left|q_{+}\left(t_{2}\right) q_{+}^{*}\left(t_{1}\right)\right| 0\right\rangle+\theta\left(t_{1}-t_{2}\right)\left\langle 0\left|q_{-}\left(t_{2}\right) q_{-}^{*}\left(t_{1}\right)\right| 0\right\rangle= \\
& =\frac{1}{2 m \omega}\left(\theta\left(t_{2}-t_{1}\right) e^{-i \omega\left(t^{\prime}-t\right)}+\theta\left(t_{1}-t_{2}\right) e^{i \omega\left(t^{\prime}-t\right)}\right) .
\end{aligned}
$$




\subsection{The inconsistency of the former standard treatment of microcausality}

The former standard formulation of the QFT was based on the microcausality condition used in the classical theory of relativistic fields. Based on the absence of superluminal signals, it was argued that field measurements at a point $x$ do not affect measurements at a point beyond the light cone .

In quantum mechanics, this meant the simultaneous measurability of field variables at these points, which was expressed in the requirement that the field operators commute (or anticommute). The commutators of bosonic fields should therefore be odd functions of the time interval disappearing behind the light cone, and this property was considered as an expression of microcausality and locality in the QFT $[9,10]$.

The commutator of the complex scalar field disappeared behind the light cone due to the replacement $\left[a_{k-}, a_{k^{\prime}-}^{*}\right]=\left[b_{k_{+}}^{*}, b_{k^{\prime}+}\right]=-\left[b_{k^{\prime}+}, b_{k_{+}}^{*}\right]$ providing the desired minus sign:

$$
\begin{aligned}
& {\left[\phi\left(x^{\prime}\right), \phi^{*}(x)\right]=\sum_{k, k^{\prime}} e^{i\left(\mathbf{k}^{\prime} \mathbf{x}^{\prime}-\mathbf{k x}\right)}\left(\left[a_{k+}, a_{k^{\prime}+}^{*}\right] e^{-i\left(\omega_{k^{\prime}} t^{\prime}-\omega_{k} t\right)}-\left[b_{k+}, b_{k^{\prime}+}^{*}\right] e^{i \omega_{k}\left(t^{\prime}-t\right)}\right)=} \\
& =-2 i \sum_{k} e^{i \mathbf{k}\left(\mathbf{x}^{\prime}-\mathbf{x}\right)} \sin \left[\omega_{k}\left(t^{\prime}-t\right)\right]=-i D\left(x^{\prime}-x\right),
\end{aligned}
$$

where $D\left(x^{\prime}-x\right)=0$ is the Pauli-Jordan function vanishing at $\left(x^{\prime}-x\right)^{2}>0$. This was considered as an evidence of the strict fulfillment in QFT of the classical microcausality condition and was widely used in various applications.

At the same time, the causal propagator for field quanta, i.e. the vacuum average from chronological products, did not disappear behind the light cone:

$$
\begin{aligned}
& \left\langle 0\left|T_{+}\left[\phi\left(x^{\prime}\right) \phi^{*}(x)\right]\right| 0\right\rangle= \\
& =\sum_{k}\left[\theta\left(t^{\prime}-t\right) e^{-i \omega_{k}\left(t^{\prime}-t\right)}+\theta\left(t-t^{\prime}\right) e^{i \omega_{k}\left(t^{\prime}-t\right)}\right] e^{i \mathbf{k}\left(\mathbf{x}^{\prime}-\mathbf{x}\right)}=i D_{c}\left(x^{\prime}-x\right),
\end{aligned}
$$

where $D_{c}\left(x^{\prime}-x\right) \neq 0$ at $\left(x^{\prime}-x\right)^{2}>0$. This meant that in QFT superluminal quantum movements take place at least at the scales of the Compton length of quanta. This is not surprising, since in this region the single-particle approximation is no longer applicable, and in a multi-particle system, where virtual pairs are present, such movements are effectively realized.

But if the propagator provides superlight signals (although in a soft form, that is, in a small neighborhood), then the initial position of classical causality about the complete absence of such signals is no longer valid. Namely, the measurement at a point $x$ can affect the measurement at a point $x^{\prime}$ even $\left(x^{\prime}-x\right)^{2}>0$ by means of virtual quanta carried by a causal propagator (101).

Thus, in the standard treatment of the QFT, on the one hand, there are superluminal signals carried by a causal propagator, and on the other hand, without paying any attention to this new circumstance, the complete absence of such signals in field measurements was required, stating on an already obsolete analogy with the classical theory. This means that in the treatment of microcausality, the theory appeared as internally contradicting even in the case of scalar fields.

In the case of fermion fields, the anti-commutator, having the form:

$$
\left\{\psi\left(x^{\prime}\right), \bar{\psi}(x)\right\}=\sum_{p}\left[(\not p+m) e^{-i E_{p}\left(t^{\prime}-t\right)} e^{i \mathbf{p}\left(\mathbf{x}^{\prime}-\mathbf{x}\right)}+(\not p-m) e^{i E_{p}\left(t^{\prime}-t\right)} e^{-i \mathbf{p}\left(\mathbf{x}^{\prime}-\mathbf{x}\right)}\right],
$$

can be represented through the Pauli-Jordan function: 


$$
\left\{\psi_{ \pm}\left(x^{\prime}\right), \bar{\psi}_{ \pm}(x)\right\}=\left(i \not x_{x^{\prime}}+m\right) i D\left(x^{\prime}-x\right),
$$

and it also disappears behind the light cone, so that the former definition of microcausality extends to fermions too. At $t^{\prime}=t(102)$ reduces to $\delta^{3}\left(\mathbf{x}^{\prime}-\mathbf{x}\right)$, as it should be for the equal time anticommutator (80).

Thus, the property of field (anti)commutators, which was presented as a strict manifestation of the classical microcausality condition in QFT, contradicted the property of causal propagators. The superluminal signals were excluded at measuring the fields, while at calculating the diagrams with causal propagators, such signals were taken into account as an important part of the physical picture, which shows the internal contradiction in the former treatments of microcausality and locality.

\subsection{The inconsistency of the former proof of the spin and statistics theorem}

In the previous standard formulation of QFT, the proof of the spin and statistics theorem was based on two conditions. In the particular case of free fields, this is the positive energy condition for the spinor field quanta, because of which anticommutators were introduced instead of commutators, and in the more general case of interacting fields this is a microcausality condition, understood as the disappearance of the field commutators behind the light cone $[9,10]$.

In Section 2.4, the spin field $1 / 2$, described by the Dirac equation, was quantized in the SF treatment by introducing anticommutators, assuming that this is a fermion field. But in this case, the anticommutators did not lead to the exclusion of the negative energy quanta. This means that the appearance of anticommutators in the consistent quantum field theory without zero-point energy is not related by the sign of the energy of quanta.

In the former standard formulation, the fact of linking the anticommutators with the energy sign was an artifact of the "manual" replacement of the creation (annihilation) operators of quanta of negative energy by the annihilation (creation) operators of antiparticles, which led to the zero-point energy.

As will be shown in Section 3.4, in the QFT based on the SF treatment, the former microcausality condition becomes modified, being softer, since the theory contains the effective nonlocality due to the creation and annihilation of virtual pairs within the Compton length.

In this case, commutators of the boson field behave similar to the causal propagators which do not disappear outside the light cone. This not only does not lead to the problems, but on the contrary, only restores the internal consistency of the QFT.

In this regard notice the following two facts, on which we will refer further in the question of the connection between spin and statistics. Firstly, it is a fact of the description of the integer spin quanta by the symmetric wave functions. It is evident in the system of two pairs of electrons, each of the pairs with an integer total spin, where at pairwise permutation of electrons the wave function of the system does not change its sign. This means that the first part of the spin and statistics theorem, that the integer spin particles are bosons, is proved obviously.

Secondly, it is a fact that the half-integer spin particles in the framework of the SF treatment can in principle be described by both symmetric and antisymmetric wave functions, but the experiments show that the second one is true, i.e. they are fermions. Thus, at this level of our understanding of QFT, the second part of the spin and statistics theorem, that the half-integer spin quanta are fermions, is not a theorem that can be proved, but is a postulate based on the experiments. 


\subsection{Commutators and causal propagators of fields}

The commutator of the complex scalar field is:

$$
\begin{aligned}
& {\left[\phi\left(x^{\prime}\right), \phi^{*}(x)\right]=\left[\phi_{+}\left(x^{\prime}\right), \phi_{+}^{*}(x)\right]+\left[\phi_{-}\left(x^{\prime}\right), \phi_{-}^{*}(x)\right]=} \\
& =\sum_{k}\left(e^{-i \omega_{k}\left(t^{\prime}-t\right)}+e^{i \omega_{k}\left(t^{\prime}-t\right)}\right) e^{i \mathbf{k}\left(\mathbf{x}^{\prime}-\mathbf{x}\right)}=-i D_{1}\left(x^{\prime}-x\right) .
\end{aligned}
$$

This result differs from the result of the former treatment with $D\left(x^{\prime}-x\right)(100)$. Now it appears $D_{1}\left(x^{\prime}-x\right)$, an even function of the time interval which does not disappear behind the light cone, although is concentrated mainly within the Compton length. In this respect, the commutator (104) behaves similar to the causal propagator. The implications of this behavior, in particular for determining microcausality, are discussed in the next section.

Let's construct the causal propagator for quanta of fields as for the oscillator, defining it as a symmetric chronological product of operators $\hat{T}$ with properties (94). The causal propagators for quanta then can be defined as the vacuum averages on the symmetric chronological products of the field operators at two spacetime points $\left\langle 0\left|\hat{T}\left[\phi\left(x^{\prime}\right) \phi^{*}(x)\right]\right| 0\right\rangle$ and $\left\langle 0\left|\hat{T}\left[\phi^{*}\left(x^{\prime}\right) \phi(x)\right]\right| 0\right\rangle$. As in the case of the oscillator, they take the form:

$$
\begin{aligned}
& \left\langle 0\left|\hat{T}\left[\phi\left(x^{\prime}\right) \phi^{*}(x)\right]\right| 0\right\rangle=\left\langle 0\left|\hat{T}\left[\phi^{*}(x) \phi\left(x^{\prime}\right)\right]\right| 0\right\rangle= \\
& =\left\langle 0\left|\phi_{+}\left(x^{\prime}\right) \phi_{+}^{*}(x)\right| 0\right\rangle \theta\left(t^{\prime}-t\right)+\left\langle 0\left|\phi_{-}\left(x^{\prime}\right) \phi_{-}^{*}(x)\right| 0\right\rangle \theta\left(t-t^{\prime}\right)= \\
& =\sum_{k}\left[\theta\left(t^{\prime}-t\right) e^{-i \omega_{k}\left(t^{\prime}-t\right)}+\theta\left(t-t^{\prime}\right) e^{i \omega_{k}\left(t^{\prime}-t\right)}\right] e^{i \mathbf{k}\left(\mathbf{x}^{\prime}-\mathbf{x}\right)}=i D_{c}\left(x^{\prime}-x\right),
\end{aligned}
$$

Thus, we come to the standard causal SF propagator, which does not disappear behind the light cone. Analogously can be derived the causal propagators for vector and tensor fields and they are the same as the standard causal propagators for these fields.

The causal propagator for fermions also is defined as the vacuum matrix element on a symmetric chronological product and also coincides with the standard causal propagator:

$$
\begin{aligned}
& i S_{c}\left(x^{\prime}-x\right)_{i j}=\left\langle 0\left|\hat{T}\left[\psi_{i}\left(x^{\prime}\right) \bar{\psi}_{j}(x)\right]\right| 0\right\rangle=-\left\langle 0\left|\hat{T}\left[\bar{\psi}_{j}(x) \psi_{i}\left(x^{\prime}\right)\right]\right| 0\right\rangle= \\
& =\left\langle 0\left|\psi_{i+}\left(x^{\prime}\right) \bar{\psi}_{j+}(x)\right| 0\right\rangle \theta\left(t^{\prime}-t\right)+\left\langle 0\left|\psi_{i-}\left(x^{\prime}\right) \bar{\psi}_{j-}(x)\right| 0\right\rangle \theta\left(t-t^{\prime}\right)= \\
& =\sum_{p}\left[\theta\left(t^{\prime}-t\right)(\not p+m) e^{-i E_{p}\left(t^{\prime}-t\right)} e^{i \mathbf{p}\left(\mathbf{x}^{\prime}-\mathbf{x}\right)}+\theta\left(t-t^{\prime}\right)(\not p-m) e^{i E_{p}\left(t^{\prime}-t\right)} e^{-i \mathbf{p}\left(\mathbf{x}^{\prime}-\mathbf{x}\right)}\right]_{i j} .
\end{aligned}
$$

\subsection{The consistency of the treatment of microcausality in the new method}

As was shown in Section 3.3, the former definition of microcausality, declared as the disappearance of commutators or anti-commutators of fields behind the light cone, contradicted to the properties of causal propagators.

The new quantization method, described in the paper, leads to even commutators for boson fields. They do not disappear behind the light cone, but quickly decline outside the Compton length. In this sense, they behave similar to the causal propagators.

From the physical point of view, this means that behind the light cone the classical conditions of locality and relativistic causality are fulfilled not strictly, but approximately because of the correlation of the field functions on the Compton length scales. Therefore, they are performed as better, as larger the distance compared with this characteristic scale of the effective non-locality in the many-particle system. 
Thus, the new method leads to the revision of the classical microcausality conditions towards their softening for quantum fields.

\subsection{Interacting fields and diagram technique}

At analyzing the consequences of the new quantization method for interacting fields, we first note some differences with the former diagram technique, and then select the part that remains valid in the new method.

If several operators of the same field are present at the interaction vertices, now it is necessary to take into account the fact that not all of these operators mutually (anti) commute, for example, $\phi(x) \phi^{*}(x) \neq \phi^{*}(x) \phi(x)$. Some consequences of this for the diagram technique and observables will be discussed in forthcoming publications.

In special cases of diagrams describing known processes and loop corrections, the operators of the same field at each vertex usually correspond to different states and therefore mutually (anti) commute, as was assumed in the former diagram technique.

The formal difference of the new method is the introduction of a time-symmetrical chronological ordering, but this leads to the standard causal propagators. Therefore, this difference was effectively taken into account in the former diagram technique at constructing the causal propagators, which is why its results coincide with the new method.

The external lines of the diagrams of the standard treatment of QFT were based in fact of the representation of antiparticles as in the SF treatment, and therefore, there are no differences in this respect either.

Since the propagators and external lines in the new and former methods are the same, the whole diagram technique will not essentially change and therefore the new method in fact legitimizes the application of the standard diagram technique, decreasing the number of its recipes only.

\section{The consistent transition from the SF treatment to the antiparticles}

The SF treatment, where the description is in terms of particles of two signs of energy and there are no antiparticles, is very convenient and sufficient for a consistent description of most of observed phenomena in particle physics and for this reason it is widely used. But for completeness of the physical picture, it is also necessary to know that from this somewhat formal picture it is always possible to go over to the picture of positive energy particles and antiparticles really observed in experiments. This transition must be carried out correctly, since the interchanging of the initial and final states, necessary for such a transition, is not a simple operation, especially for the operator products, and it requires a certain set of necessary steps.

The standard transition procedure was just an example of inaccuracy in this case, which became one of the reasons for the failure of the entire standard treatment of QFT. The procedure was reduced to manually substituting the creation (annihilation) operators of antiparticles instead of the annihilation (creation) operators of negative energy particles directly in the field operators, regardless in what of products these field operators appear. Such a simplified and naive approach then generated the divergent zero-point energies in the free Hamiltonians, and turned out to be negative (!) for fermions, which then required an artificial normal ordering recipe, including in the case of interaction terms.

Below two consecutive procedures for the transition from the SF treatment to the picture of antiparticles will be described. The first, based on the charge conjugation $(C)$ symmetry, is simple and convenient to apply to the equal time bilinear products of field operators, such as free Hamiltonians and currents. The second one, based on the widely 
used in practice form of the SF treatment - crossing symmetry, is more universal and can be applied to the propagators and interaction operators too.

\subsection{Transition to the antiparticles by means of charge conjugation symmetry}

Simplest procedure based on the $C$-symmetry, allowing one to exclude the operators of negative energy quanta from the operators of observables, will be shown on the example of an oscillator with complex coordinates.

Such an oscillator with the Hamiltonian and the charge operator (37):

$$
H=\omega\left(a_{+}^{*} a_{+}-a_{-}^{*} a_{-}\right), \quad Q=a_{+}^{*} a_{+}+a_{-}^{*} a_{-}, \quad\left[a_{ \pm}, a_{ \pm}^{*}\right]=1,
$$

is $C$-symmetric, i.e. at $C$ - conjugation $H$ is invariant, while $Q$ changes a sign:

$$
H^{c} \equiv C H C^{-1}=H, \quad Q^{c} \equiv C Q C^{-1}=-Q .
$$

The ladder operators, charge conjugate to the ordinary ones, are defined as:

$$
b_{ \pm}=C a_{ \pm} C^{-1}, \quad b_{ \pm}^{*}=C a_{ \pm}^{*} C^{-1}, \quad\left[b_{ \pm}, b_{ \pm}^{*}\right]=1,
$$

and the charge conjugate Hamiltonian and charge are expressed through them as:

$$
H^{c}=\omega\left(b_{+}^{*} b_{+}-b_{-}^{*} b_{-}\right), Q^{c}=b_{+}^{*} b_{+}+b_{-}^{*} b_{-} .
$$

Further, using the $C$-symmetry conditions (108), we replace the operator products $a_{-}^{*} a_{-}$and $b_{-}^{*} b_{-}$for the negative-energy states with the operator products $b_{+}^{*} b_{+}$and $a_{+}^{*} a_{+}$ for the positive-energy states. For this we write the conditions (108) explicitly:

$$
\begin{aligned}
& H=\omega\left(a_{+}^{*} a_{+}-a_{-}^{*} a_{-}\right)=\omega\left(b_{+}^{*} b_{+}-b_{-}^{*} b_{-}\right)=H^{c}, \\
& Q=a_{+}^{*} a_{+}+a_{-}^{*} a_{-}=-\left(b_{+}^{*} b_{+}+b_{-}^{*} b_{-}\right)=-Q^{c}
\end{aligned}
$$

and we get two operator relations for four types of products:

$$
\begin{aligned}
& a_{+}^{*} a_{+}-a_{-}^{*} a_{-}=b_{+}^{*} b_{+}-b_{-}^{*} b_{-}, \\
& a_{+}^{*} a_{+}+a_{-}^{*} a_{-}=-b_{+}^{*} b_{+}-b_{-}^{*} b_{-} .
\end{aligned}
$$

Further, by adding and subtracting these two relations, we obtain two operator identities:

$$
a_{-}^{*} a_{-}=-b_{+}^{*} b_{+}, \quad b_{-}^{*} b_{-}=-a_{+}^{*} a_{+},
$$

where the second identity is the charge-conjugated form of the first one. They allow us to exclude $a_{-}^{*} a_{-}$and $b_{-}^{*} b_{-}$from the operators of observables (107) and (110):

$$
\begin{aligned}
& H=H^{c}=\omega\left(a_{+}^{*} a_{+}+b_{+}^{*} b_{+}\right), \\
& Q=-Q^{c}=a_{+}^{*} a_{+}-b_{+}^{*} b_{+} .
\end{aligned}
$$

Thus, the $C$-symmetry conditions allow us to transform the SF picture to the antiparticles picture.

Notice that the operator relations, including identities (113), have the meaning of equality of means over the corresponding states and, for completeness, it is necessary to make charge conjugations of the states over which these averages are taken.

\subsection{Transition to antiparticles by using crossing symmetry}

In the former standard treatment of QFT, the operators of negative-energy particles $a_{-k}$ and $a_{-k}^{*}$ were replaced "manually" by the operators of positive-energy antiparticles directly in the field operators: 


$$
a_{-k}^{*} \rightarrow b_{k}, a_{-k} \rightarrow b_{k}^{*}
$$

At the same time, the possibility of such a replacement in the products of operators was not proved, but was naively implied as an obvious consequence of (115).

However, in the SF treatment, as well as in one of its practically used in particle physics forms - the crossing symmetry, such a transition involves replacing the initial and final states, which leads to non-trivial consequences for the operator products. Therefore, carefulness and more accurate definitions are required here, in particular, all replacements of the particle operators $a_{-k}, a_{-k}^{*}$ and their products to the antiparticle operators $b_{k}^{*}, b_{k}$ and their products must be consistent with the crossing symmetry conditions.

Crossing symmetry makes it possible to easily obtain the amplitudes of several other "related" processes from the expression for the amplitude of one a process. Due to this symmetry, a particle in the initial (final) state with a 4-momentum $+p_{\mu}$ can be transferred to the final (initial) state with a 4-momentum $-p_{\mu}$ and, after changing the direction of the time evolution to the opposite, be considered as a description of the antiparticle:

$$
A+B \rightarrow C+D, \quad A+\bar{C} \rightarrow \bar{B}+D, \quad A+\bar{D} \rightarrow C+\bar{B} .
$$

Thus, a crossing operation, consisting of turning a line around a vertex in a diagram with transition into another light cone, changing the sign of the 4-momentum and the direction of time evolution, turns the particle into the antiparticle (and vice versa).

In the crossing transformation, the change in the sign of the 4-momentum of the particle and the change in the sign of the time interval cancel each other, which leaves the sign of the energy of the resulting antiparticle positive. In this case, the particles move in the direction of the arrow in the diagram, and the antiparticle in the opposite direction. Thus, the line of the particle directed to the past corresponds to the antiparticle going to opposite side, i.e. to the future.

Notice, that the SF treatment, and hence the crossing transformation, refers to a single line in the Feynman diagram, independently of other lines, which distinguishes it from the CPT symmetry, transforming the whole diagram.

As an example, we consider the relationship between the scattering amplitudes of electrons and positrons in a Coulomb field. The positron transition matrix element:

$$
S_{f i}=i e \int d^{4} x\left\langle-p_{i}, s_{i}\left|\bar{\psi}(x) \gamma^{0} \psi(x)\right|-p_{f}, s_{f}\right\rangle A_{0}(x) .
$$

at substituting the field operators (78) takes the form:

$$
S_{f i}=-\sum_{p, s}\left\langle-p_{i}, s_{i}\left|b_{-p, s}^{+} b_{-p, s}\right|-p_{f}, s_{f}\right\rangle \tilde{S}_{f i},
$$

where $\tilde{S}_{f i}$ is all other factors. At the same time, after crossing transformation of the amplitude describing the electron scattering, turning it into an amplitude for the positron scattering, we get:

$$
S_{f i}=\sum_{p, s}\left\langle p_{f}, s_{f}\left|d_{p, s}^{+} d_{p, s}\right| p_{i}, s_{i}\right\rangle \tilde{S}_{f i} .
$$

The comparison of these two expressions, (118) and (119), gives:

$$
-\left\langle-p_{i}, s_{i}\left|b_{-p, s}^{+} b_{-p, s}\right|-p_{f}, s_{f}\right\rangle=\left\langle p_{f}, s_{f}\left|d_{p, s}^{+} d_{p, s}\right| p_{i}, s_{i}\right\rangle
$$

which is the matrix form of the operator relation (113) for the case of fermions. 
Matching of the quantization rules with such a fundamental symmetry of particle physics as crossing symmetry leads to a number of modifications that, together with those discussed above, lead to the crossing symmetric quantization. The consequences for interacting fields, including clarifications in the definitions of causal propagators, the ordering rules for operator products, and the diagram technique will be discussed later.

\section{On the consequences of the lack of zero-point energy of fields}

\subsection{The experiments confirmed the lack of the zero-point energy of fields}

The quantum fluctuations of fields of real sources, such as electrons and protons, lead to the set of observed effects. The predictions of QFT with such real sources are consistent with experiments and often the accuracy of this agreement is unprecedented.

In particular, the Lamb shift is determined by the interaction term $H_{I}^{(r)}=\mathbf{j} \cdot \mathbf{A}_{(r)}$, where $\mathbf{j}$ is the current of electrons or protons, and $\mathbf{A}_{(r)}$ describes the virtual photons, the excited states of electromagnetic field. The latter are not relate to the hypothetical external vacuum fields $\mathbf{A}_{(0)}$, associated by the zero-point vacuum energy $H_{0}^{(0)}$. The experiments showed that the observed value of the Lamb shift $\Delta E_{I}^{\exp }$ is completely explained by the contribution $\Delta E_{I}^{(r)}$ of the virtual photons from real sources:

$$
\Delta E_{I}^{\mathrm{exp}}=\Delta E_{I}^{r},
$$

But in the former standard treatment of QFT, the zero-point vacuum fluctuations additionally contribute to the Lamb shift. In this case, the zero-point energy of the electromagnetic field $H_{0}^{(0)}$, which was inevitable there, should have been generated by the fluctuating vacuum fields $\mathbf{E}_{(0)}, \mathbf{B}_{(0)}$ :

$$
H_{0}^{(0)}=2 \int d^{3} k \frac{1}{2} \omega_{k}=\int d^{3} x \frac{1}{2}\left(\mathbf{E}_{(0)}^{2}+\mathbf{H}_{(0)}^{2}\right)
$$

and the total energy would be contain a contribution from the additional interaction term with these vacuum fields $H_{I}^{(0)}=\mathbf{j} \cdot \mathbf{A}_{(0)}$, where $\mathbf{E}_{(0)}, \mathbf{B}_{(0)}$ are the strengths of the field $\mathbf{A}_{(0)}[9,10]$. Since this additional interaction energy $H_{I}^{(0)}$ enters to the interaction Hamiltonian additively with the interaction energy $H_{I}^{(r)}$ of the same particles with the fields of the real sources $\mathbf{A}_{(r)}$, the contribution of the zero-point fluctuations $\Delta E_{I}^{(0)}$ should be added to the contribution of the real sources $\Delta E_{I}^{(r)}$.

Since both contributions are of the same order (which is why they were usually confused with each other), in fact the predicted energy shifts would be doubled:

$$
\Delta E_{I}=\Delta E_{I}^{(0)}+\Delta E_{I}^{(r)} \approx 2 \Delta E_{I}^{r} \text {. }
$$

The observations indicate the existence of only a single contribution, which in the conventional scientific methodology should be attributed to the contribution of the real sources $\Delta E_{I}^{r}$, since this contribution cannot be ignored in any case.

In the case of the Casimir effect, the observed effect is completely explained by the contribution of the fields of other kind of real sources - by the fluctuating radiation field of crystal atoms. It is well known that the theory of Van der Waals forces successfully describes the Casimir effect, including the temperature dependence, through the radiation 
of real sources - atoms - at their vibrations in the crystal, including the vibrations at the "zero-point temperature" of the crystal. Since the contribution of the purely vacuum fields $H_{I}^{(0)}$ has the same order and it should be summed with the contribution $H_{I}^{(r)}$ of the radiation field of atoms, the former treatment with the zero-point energy in this case also gives doubled value of the Casimir effect than it is really observed.

Thus, the contribution of the real sources, which cannot be ignored, completely explains the experiments in the case of both effects. This means that the existing experiments exclude the contribution of the zero-point vacuum fluctuations and, therefore, only the new method of quantization on the basis of the SF treatment, where they are absent, is in agreement with these experiments.

However, by the irony of history, a myth was created about the same experiments that they allegedly confirmed the opposite statement that the zero-point vacuum energy was clearly manifested in them. This myth was then widely promoted in the literature, including the well-known textbooks of QFT. In fact, it is based on the logical trick used in the manipulative propaganda, the mean of which is that at first one explains the real phenomenon by the desired unrealistic reason, artificially excluding from the consideration a real reason. And since one must explain the same phenomenon by the real reason too, one excludes from the consideration the unrealistic reason. As a result, a false belief becomes created that the unreal reason is acceptable in the equal rights by the real one and, therefore, unreal is also real, since it is also "confirmed" by the observations.

Note that this case of applying "non-traditional" logic in science is an example of the phenomenon of "doublethink", the "ability to adhere to two opposing beliefs at the same time", well-known in psychology. In the traditional scientific methodology, if a phenomenon is supposed to have two simultaneously influencing causes, then the consequences of their influence should also be taken into account simultaneously. If, however, the phenomenon is completely explained only by one of the reasons, the existence of which is beyond doubt, then the second reason should be considered as non-existent and refuted. In our case, the reason that cannot be ignored, and which is sufficient to explain the observations, is the field of real sources, while the mythical "alternative", without which everything is already explained, is the influence of the zero-point fluctuations of fields.

\subsection{Partial solution of the cosmological constant problem}

As it is shown by observations, the value of the cosmological constant is practically disappearing and the new quantization on the basis of the SF treatment naturally explains this observational fact, in the sense of the lack of contribution from the vacuum of free fundamental fields.

However, this fact was mysterious in the former treatment of QFT with a very large contribution of the zero-point vacuum energy. All attempts to cancel this contribution in the framework of this form of QFT only make worse the situation, requiring additional unrealistic hypotheses.

It is important that attempts to eliminate the zero-point energy by artificial methods, such as «normal ordering» or shifting the energies, was not acceptable due to the existence of gravity and this fact was well known. In this regard, the Standard Model, based on the former quantization method, was inconsistent, and this fact was clearly recognized.

Therefore, the lack of the zero-point vacuum energy of the fields at quantization of them by the new method indicates an internal self-consistency and reliability of the physical ideas and principles which lies on the basis of the entire relativistic quantum theory.

At the same time, this only excludes the contribution of the vacuum of free fields and therefore does not mean the final solution of the cosmological constant problem. Other 
possible contributions, such as contributions from the field condensates, the loop contributions, etc. are beyond the competence of the theory of free fields and require consideration of the theory of interacting fields.

Notice, that in the theory of interacting fields, the new method leads to automatic normal ordering of many operator products, which eliminates the diverging loop contributions to the vacuum energy. This fact, therefore, solves the cosmological constant problem also in the part concerning the contribution of the vacuum of interacting fields, at least in the framework of the perturbation theory.

\subsection{Supersymmetry does not solve the zero-point energy problem}

The possible existence of supersymmetry, symmetry between bosons and fermions, which allows to solve the problems with divergences in field theory and the unification problem in particle physics, was one of the most inspiring and systematically developed hypotheses over the past half century [8].

There were three expectations for this new symmetry: mutual cancellation of the zero-point energies of bosons and fermions, partial cancellation of the loop diagrams divergences, and the creating of successful models of unification. Here it will be discussed only the first of these hopes.

The idea about possible cancellation of the zero-point energies of bosons and fermions follows from the fact that, at the standard quantization of fermion fields there appears not an ordinary zero-point energy, but a zero-point energy of negative sign!

However, at quantization of the relativistic fields in the new method based on the SF treatment a zero-point energy does not appear for both bosons and fermions and thus the initial idea of the supersymmetry appears to be realized naturally, without such radical hypothesis.

Thus, one of the three main arguments in favor of supersymmetry, associated with the zero-point energies, turned out to be irrelevant.

\section{Finite quantum theory of strings in the SF treatment}

\subsection{Inconsistency of the string theories with zero-point energy}

The most radical of the widely promoted unification models was the hypothesis that the fundamental objects of physics are not localized particles and continuous fields, but one-dimensional objects - strings. In theories of relativistic strings, it appears a critical dimension of space-time: $D=26$ for a bosonic string, $D=10$ for a fermionic string and a superstring. It is the key property underlying almost all the achievements of the string theories.

In Appendix B it is shown that the critical dimension is a consequence of the presence of the zero-point energy of string modes and the need for normal ordering of the operators of observables. In this case, the critical dimensions follow from the requirement to cancel anomalies arising from the residual contribution of zero-point energies of string modes after their artificial "regularization".

But the fact of the appearance of diverging zero-point energy means the inconsistency of such unification theories. This is because the "regularization" was justified as a temporary procedure for semi-phenomenological theories as an approximation of unknown behavior at short distances. But it is unacceptable for a fundamental theory that claims to be a correct description of physics at these small distances, where gravity is not only strong, but even dominates.

As it will be shown below, in one of the models of the relativistic string, quantized in accordance with the SF treatment, the zero-point energy does not arise, and therefore 


\section{Consistent quantization in the Stueckelberg-Feynman treatment}

there will be no critical dimension. Such string theory is physically consistent, but it can no longer solve the problems of unification.

If someone tries to fix a certain dimension of space-time by using other reasons even in the absence of a critical dimension, by introducing special symmetries, then even this case such models appear as less suitable than models based on local primary objects. Really, the introduction of one-dimensional objects with a continuously distributed energy density along their length, as well as the nature of stresses along their length, causes a number of new problems, which were present in the string theories, but not analyzed properly. Obviously, it is almost impossible to avoid the divergences, that are still hidden, due to the structure of any one-dimensional object, especially for objects of higher dimension. This shows a high degree of artificiality and internal contradictions in the models with nonlocal fundamental objects.

\subsection{Consistent quantization of strings without zero-point energy and anomalies}

The lack of the zero-point energy of string modes leads to the string theory without conformal anomaly, since the zero-point energy was a source of this anomaly. Due to the lack of the anomaly, there is no reason to fix the critical dimension of spacetime. For this reason, below it will be formulated the simplest string model without zero-point energy.

Consider the standard action for the bosonic string [8]:

$$
S=-\frac{T}{2} \int d^{2} \sigma \sqrt{h} h^{\alpha \beta} \partial_{\alpha} X^{\mu} \partial_{\beta} X_{\mu}
$$

where $h^{\alpha \beta}=h^{\alpha \beta}(\tau, \sigma), h=\left|\operatorname{det} h^{\alpha \beta}\right|, X^{\mu}=X^{\mu}(\tau, \sigma)$. The symmetries of the string allow us to take the 2-metric as $h_{\alpha \beta}(\tau, \sigma)=\eta_{\alpha \beta}=\operatorname{diag}(1,-1)$, after which Eq. (124) gives:

$$
\begin{aligned}
S=-\frac{T}{2} \int d^{2} \sigma \partial_{\alpha} X^{\mu} \partial^{\alpha} X_{\mu} & =-\frac{T}{2} \int d^{2} \sigma\left(\partial_{\alpha} X_{R}^{\mu} \partial^{\alpha} X_{\mu R}+\partial_{\alpha} X_{L}^{\mu} \partial^{\alpha} X_{\mu L}\right), \\
X^{\mu}(\tau, \sigma) & =X_{R}^{\mu}(\tau-\sigma)+X_{L}^{\mu}(\tau+\sigma) .
\end{aligned}
$$

Here the right $X_{R}^{\mu}$ and left $X_{L}^{\mu}$ modes of a closed string are the solutions of the equations of motion:

$$
\partial_{\alpha} \partial^{\alpha} X^{\mu}=\left(\partial_{\tau}^{2}-\partial_{\sigma}^{2}\right) X^{\mu}=0
$$

The coordinates of these modes can be combined into one complex coordinate:

$$
Y^{\mu}=\frac{1}{\sqrt{2}}\left(X_{R}^{\mu}+i X_{L}^{\mu}\right), \quad Y^{\mu^{*}}=\frac{1}{\sqrt{2}}\left(X_{R}^{\mu}-i X_{L}^{\mu}\right) .
$$

In terms of this coordinate, the action and the energy-momentum tensor take the form:

$$
S=-\frac{T}{2} \int d^{2} \sigma\left(\partial_{\alpha} Y_{\mu}^{*} \partial^{\alpha} Y^{\mu}+\partial^{\alpha} Y^{\mu} \partial_{\alpha} Y_{\mu}^{*}\right) .
$$

At the classical level the string variables are ordinary functions and are therefore permutable, so that the integrand in (129) is equivalent to the expression with the minimal Lagrangian:

$$
S=-T \int d^{2} \sigma \partial_{\alpha} Y_{\mu}^{*} \partial^{\alpha} Y^{\mu}
$$

We will consider the classical action function in (130) as the starting point for further quantization. 
In the SF treatment, firstly, instead of a superposition of modes $\tau \pm \sigma$, moving right or left, each with positive and negative energies, one of the modes is selected as the basic one, for example, the right one with positive energy $Y_{+}^{\mu}(\tau-\sigma)$, moving forward in time only. Secondly, the left positive mode $Y_{+}^{\mu}(\tau+\sigma)$ is described as the right mode, but with negative energy $Y_{-}^{\mu}(\tau-\sigma)$ and moving only backward in time. Thirdly, the action function in (130) should be rewritten by taking into account these two points as:

$$
S=-T \int d \sigma\left[\theta\left(\tau_{1}-\tau_{0}\right) \int_{\tau_{0}}^{\tau_{1}} d \tau \partial_{\alpha} Y_{+}^{*} \cdot \partial^{\alpha} Y_{+}-\theta\left(\tau_{0}-\tau_{1}\right) \int_{\tau_{0}}^{\tau_{1}} d \tau \partial_{\alpha} Y_{-}^{*} \cdot \partial^{\alpha} Y_{-}\right] .
$$

The equations of motion (131) and the boundary conditions for closed strings:

$$
Y^{\mu}(\sigma)=Y^{\mu}(\sigma+2 \pi), \quad Y^{\mu^{*}}(\sigma)=Y^{\mu^{*}}(\sigma+2 \pi),
$$

lead to the frequency decomposition of the complex coordinate:

$$
\begin{aligned}
& Y^{\mu}(\tau-\sigma)=Y_{+}^{\mu}+Y_{-}^{\mu}=Y_{0}^{\mu}+\frac{i l}{2} \sum_{n=1}^{\infty} \frac{1}{n}\left(\alpha_{n}^{\mu} e^{-2 i n(\tau-\sigma)}+\alpha_{-n}^{\mu} e^{2 i n(\tau-\sigma)}\right), \\
& Y^{\mu^{*}}(\tau-\sigma)=Y_{+}^{\mu^{*}}+Y_{-}^{\mu^{*}}=Y_{0}^{\mu^{*}}-\frac{i l}{2} \sum_{n=1}^{\infty} \frac{1}{n}\left(\alpha_{n}^{\mu^{*}} e^{2 i n(\tau-\sigma)}+\alpha_{-n}^{\mu^{*}} e^{-2 i n(\tau-\sigma)}\right),
\end{aligned}
$$

where $l^{2}=1 / \pi T$. This also gives:

$$
\begin{aligned}
& \dot{Y}^{\mu}=\dot{Y}_{+}^{\mu}+\dot{Y}_{-}^{\mu}=\dot{Y}_{0}^{\mu}+l \sum_{n=1}^{\infty}\left(\alpha_{n}^{\mu} e^{-2 i n(\tau-\sigma)}-\alpha_{-n}^{\mu} e^{2 i n(\tau-\sigma)}\right), \\
& \dot{Y}^{\mu^{*}}=\dot{Y}_{+}^{\mu^{*}}+\dot{Y}_{-}^{\mu^{*}}=\dot{Y}_{0}^{\mu^{*}}+l \sum_{n=1}^{\infty}\left(\alpha_{n}^{\mu^{*}} e^{2 i n(\tau-\sigma)}-\alpha_{-n}^{\mu^{*}} e^{-2 i n(\tau-\sigma)}\right) .
\end{aligned}
$$

Here the times $\tau$ are multiplied only to the positive "frequencies" $n>0$ and the sums of contributions with opposite signs of energy are written separately.

Conserved currents of the coordinate translation $Y^{\mu}, Y^{\mu^{*}}$ are:

$$
P_{\alpha \pm}^{\mu}= \pm T \partial_{\alpha} Y_{ \pm}^{\mu^{*}}, \quad \partial^{\alpha} P_{\alpha \pm}^{\mu}=0 .
$$

The commutators have the form:

$$
\left[Y^{\mu}\left(\tau, \sigma^{\prime}\right), P_{\tau}^{v}(\tau, \sigma)\right]=-i \eta^{\mu v} \delta\left(\sigma-\sigma^{\prime}\right) .
$$

Substitution of (133) and (134) gives (nonzero) commutators for the operators of modes:

$$
\left[\alpha_{m}^{\mu}, \alpha_{n}^{v^{*}}\right]=\left[\alpha_{-m}^{\mu}, \alpha_{-n}^{v^{*}}\right]=-m \delta_{m n} \eta^{\mu v} .
$$

The Hamiltonian and chiral charge are defined as:

$$
H_{ \pm}= \pm T^{-1} \int_{0}^{2 \pi} d \sigma P_{\alpha \pm} \cdot P_{ \pm}^{\alpha^{*}}, \quad \Lambda_{ \pm}= \pm \int_{0}^{2 \pi} d \sigma\left(P_{\tau \pm} Y_{ \pm}-Y_{ \pm}^{*} P_{\tau \pm}^{*}\right) .
$$

The inserting of the mode decomposition gives:

$$
H=H_{+}+H_{-}=H_{0}+\sum_{n=1}^{\infty}\left(\alpha_{n}^{\mu^{*}} \alpha_{n \mu}-\alpha_{-n}^{\mu^{*}} \alpha_{-n \mu}\right),
$$




$$
\Lambda=\Lambda_{+}+\Lambda_{-}=\Lambda_{0}+\sum_{n=1}^{\infty}\left(\alpha_{n}^{\mu^{*}} \alpha_{n \mu}+\alpha_{-n}^{\mu^{*}} \alpha_{-n \mu}\right)
$$

As a result, the operators of the Virasoro algebra (see Appendix B)

$$
L_{m}=L_{m+}+L_{m-}=\sum_{n=-\infty}^{\infty} \alpha_{m+n}^{\mu^{*}} \alpha_{n \mu}
$$

are normal ordered initially, $L_{0}|0\rangle=0$ and their commutators have the form:

$$
\left[L_{m}, L_{n}\right]=(m-n) L_{m+n} .
$$

Since they do not contain anomalies or a central extension, the algebra of operators of the Lorentz group is closed also without anomalous terms.

If it is necessary, the transition to only positive energy states is carried out in one of the two methods described in Section 4, which will give $\alpha_{-n}^{\mu^{*}} \alpha_{-n \mu} \rightarrow-b_{n}^{\mu^{*}} b_{n \mu}$. As a result, (139) and (140) turn to:

$$
H=H_{0}+\sum_{n=1}^{\infty}\left(\alpha_{n}^{\mu^{*}} \alpha_{n \mu}+b_{n}^{\mu^{*}} b_{n \mu}\right), \quad \Lambda=\Lambda_{0}+\sum_{n=1}^{\infty}\left(\alpha_{n}^{\mu^{*}} \alpha_{n \mu}-b_{n}^{\mu^{*}} b_{n \mu}\right) .
$$

The operators of modes in the Hamiltonian (139) or (143) are in the normal form and there is no zero-point energy in the theory.

Thus, the theory of a bosonic string does not contain the zero-point energy and the former restrictions on the spacetime dimension $D$. A similar situation is in the theory of a fermionic string, since at the quantization according to the SF treatment there is also no zero-point energy and no anomalies.

\section{Conclusion}

In its former standard formulation, the theory of relativistic fields and strings was inconsistent, since it inevitably led to the diverging zero-point energy. In the SF treatment, where the signs of energy and directions of time evolution are the same, the zero-point energy was absent in the case of minimal Lagrangians, but there was a problem of the negative norm of states, which is mathematically unacceptable.

It is shown that at following the SF treatment, starting from the action function, the norms of all states become positive, which makes the theory mathematically correct, and the choice of minimum Lagrangians, do not leading to the zero-point energy, makes the theory consistent from a physical point of view too.

The construction of causal propagators through the time-symmetric operator of chronological ordering, directly leading to the SF causal propagator, also becomes natural and consistent.

The new quantization method is first applied to harmonic oscillators with both signs of energy, and then to relativistic fields and strings. The result was almost the same diagram technique of the standard formulation of QFT, but without a number of semi-empirical recipes of the former formalism.

The new quantization method based on the SF treatment without zero-point vacuum energy is in agreement with the existing experiments, fully explaining their results through the contributions of the fields of real sources. The lack of the zero-point energy partially solves the cosmological constant problem in the sense of the vacuum of free fields.

At a certain choice of the Lagrangian, the modes of a relativistic string do not contain the zero-point energy and, therefore, the theory is finite, but in such a consistent string 
theory there is no anomalies in the Virasoro algebra and the algebra of the Lorentz group, thus it does not have the critical dimensions of spacetime. There is also no need for supersymmetry to solving the vacuum energy problem.

A more detailed presentation of the consecutive method of quantization on the basis of the SF treatment, formulated in the paper, and its consequences is given in the book [12].

\section{Appendices.}

\section{A. Oscillator with two signs of energy in quantum mechanics}

In the SF treatment the positive energy states of harmonic oscillators should propagate only forward in time, and with negative energy - only backward in time. The Lagrangian $L$ and the Hamiltonian $H$ of the linear harmonic oscillators with two signs of the energy of an oscillating particle have the form:

$$
L_{ \pm}= \pm \frac{m}{2}\left(\dot{x}_{ \pm}^{2}-\omega_{ \pm}^{2} x_{ \pm}^{2}\right), \quad H_{ \pm}= \pm \frac{1}{2 m}\left(p_{ \pm}^{2}+m^{2} \omega_{ \pm}^{2} x_{ \pm}^{2}\right)
$$

Here $x_{ \pm}$and $p_{ \pm}= \pm m \dot{x}_{ \pm}$are the real-valued coordinate and momentum of the particles. The wave equations then take the form (in this section $\hbar \neq 1$ ):

$$
i \hbar \frac{\partial \psi_{ \pm}}{\partial t}= \pm \frac{1}{2 m}\left(-\hbar^{2} \frac{\partial^{2}}{\partial x_{ \pm}^{2}}+(m \omega)^{2} x_{ \pm}^{2}\right) \psi_{ \pm} .
$$

The expansion over stationary states with energies $E_{n \pm}= \pm E_{n}$, where $E_{n}=\left|E_{n \pm}\right|$, are:

$$
\psi_{ \pm}\left(x_{ \pm}, t\right)=\sum_{n=0}^{\infty} \psi_{n_{ \pm}}\left(x_{ \pm}\right) e^{-i E_{n_{ \pm}} t / \hbar}=\sum_{n=0}^{\infty} \psi_{n_{ \pm}}\left(x_{ \pm}\right) e^{\mp i E_{n} t / \hbar}, n_{ \pm}=0,1,2, \ldots
$$

Here $\psi_{n_{ \pm}}\left(x_{ \pm}\right)$are spatial parts of the wave functions normalized as:

$$
\int_{-\infty}^{\infty} d x_{ \pm} \psi_{n \pm}^{2}\left(x_{ \pm}\right)=1
$$

Substituting (146) into the wave equation (145) gives the similar equations:

$$
\frac{d^{2} \psi_{n \pm}}{d x^{2}}+\left(k_{n}^{2}-\lambda^{2} x_{ \pm}^{2}\right) \psi_{n \pm}=0, \quad k_{n}^{2}=\frac{2 m E_{n}}{\hbar^{2}}, \quad \lambda=\frac{m \omega}{\hbar} .
$$

Their solutions are expressed through Hermite polynomials $H_{n}$ :

$$
\psi_{n \pm}\left(x_{ \pm}\right)=\frac{(m \omega / \pi \hbar)^{1 / 4}}{\left(2^{n} n !\right)^{1 / 2}} e^{-\frac{m \omega}{2 \hbar} x_{ \pm}^{2}} H_{n}\left[(m \omega / \hbar)^{1 / 2} x_{ \pm}\right],
$$

and the eigenvalues for $k_{n}^{2}$ and $E_{n}$ are equal to:

$$
k_{n}^{2}=\lambda(2 n+1), \quad E_{n}=\hbar \omega\left(n_{ \pm}+1 / 2\right) .
$$

This gives for the energy levels of a system of two types of oscillators:

$$
E_{n \pm}= \pm E_{n}= \pm \hbar \omega\left(n_{ \pm}+1 / 2\right) .
$$

Notice that the sign of energy is determined only by the sign in Hamiltonian (144), into which the frequency enters quadratically.

The equations of motion, following from (144), and their solutions have the form:

$$
\ddot{x}_{ \pm}+\omega^{2} x_{ \pm}=0 \text {. }
$$


1. Consistent quantization in the Stueckelberg-Feynman treatment

$$
\begin{gathered}
x_{ \pm}=\frac{1}{\sqrt{2 m \omega / \hbar}}\left(a_{ \pm} e^{\mp i \omega t}+a_{ \pm}^{*} e^{ \pm i \omega t}\right), \quad p_{ \pm}=\frac{-i m \omega}{\sqrt{2 m \omega / \hbar}}\left(a_{ \pm} e^{\mp i \omega t}-a_{ \pm}^{*} e^{ \pm i \omega t}\right), \\
a_{ \pm}=\frac{e^{ \pm i \omega t}}{\sqrt{2 m \omega \hbar}}\left(m \omega x_{ \pm}+i p_{ \pm}\right), \quad a_{ \pm}^{*}=\frac{e^{\mp i \omega t}}{\sqrt{2 m \omega \hbar}}\left(m \omega x_{ \pm}-i p_{ \pm}\right) .
\end{gathered}
$$

At quantization, the commutators $\left[x_{ \pm}, p_{ \pm}\right]=i \hbar$ lead to:

$$
\left[a_{ \pm}, a_{ \pm}^{*}\right]=1, \quad\left[a_{ \pm}, a_{ \pm}\right]=\left[a_{ \pm}^{*}, a_{ \pm}^{*}\right]=0 .
$$

Notice, that operators with different signs of energy act in different space of states and mutually commute. The wave function of ground states is also similar:

$$
\left|0_{ \pm}\right\rangle=\psi_{0}\left(x_{ \pm}\right)=(m \omega / \pi \hbar)^{1 / 4} e^{-(m \omega) x_{ \pm}^{2} / 2 \hbar}, a_{ \pm}\left|0_{ \pm}\right\rangle=0 .
$$

The wave functions of excited states of both kind of oscillators $\left|n_{ \pm}\right\rangle$are built as:

$$
a_{ \pm}\left|n_{ \pm}\right\rangle=\sqrt{n_{ \pm}}\left|n_{ \pm}-1\right\rangle, \quad a_{ \pm}^{*}\left|n_{ \pm}\right\rangle=\sqrt{n_{ \pm}+1}\left|n_{ \pm}+1\right\rangle, \ldots
$$

where $n_{ \pm}=0,1,2, \ldots$ are eigenvalues of $N_{ \pm}=a_{ \pm}^{*} a_{ \pm}$, the numbers of two types of quanta. The ladder operators in this case act on the wave functions in the representation of occupation numbers. In this case the Hamiltonian (144) takes the form:

$$
H_{ \pm}= \pm \hbar \omega\left(a_{ \pm}^{*} a_{ \pm}+1 / 2\right) \text {. }
$$

Thus, the theory of a harmonic oscillator, expanded with the inclusion of the negative energy states, is internally self-consistent, if the evolution backward in time of the negative energy states is taken into account.

\section{B. Zero-point energy as a source of anomalies and critical dimensions of strings}

The action for a boson string (125) leads to the traceless energy-momentum tensor and the constraint equations $\left(\dot{X}=\partial_{\tau} X, X^{\prime}=\partial_{\sigma} X\right)$ [8]:

$$
\begin{gathered}
T_{\alpha \beta}=-\frac{2}{T \sqrt{h}} \frac{\delta S}{\delta h^{\alpha \beta}}=\frac{1}{2}\left(\partial_{\alpha} X \cdot \partial_{\beta} X-\frac{1}{2} \eta_{\alpha \beta} \partial_{\alpha^{\prime}} X \cdot \partial^{\alpha^{\prime}} X\right)=0 . \\
T_{00}=T_{11}=\frac{1}{2}\left(\dot{X}^{2}+X^{\prime 2}\right)=0, \quad T_{10}=T_{01}=\dot{X} \cdot X^{\prime}=0 .
\end{gathered}
$$

The equations of motion and their solutions have the form:

$$
\partial_{\alpha} \partial^{\alpha} X=\left(\partial_{\tau}^{2}-\partial_{\sigma}^{2}\right) X=0, \quad X(\tau, \sigma)=X_{R}(\tau-\sigma)+X_{L}(\tau+\sigma) .
$$

The expansion of the coordinates under the string modes, following from (161) and the boundary conditions for an open string $X^{\prime}(\tau, 0)=X^{\prime}(\tau, \pi)=0$, has the form:

$$
X^{\mu}(\tau, \sigma)=x^{\mu}+l^{2} \tau p^{\mu}+i l \sum_{n \neq 0} \frac{1}{n} \alpha_{n}^{\mu} e^{-i n \tau} \cos n \sigma
$$

where $l^{2}=1 / \pi T$. The non-zero commutators are equal to $\left(P^{v}=T \dot{X}^{v}\right)$ :

$$
\begin{gathered}
{\left[X^{\mu}(\tau, \sigma), P^{v}\left(\tau, \sigma^{\prime}\right)\right]=i \eta^{\mu v} \delta\left(\sigma-\sigma^{\prime}\right),} \\
{\left[\alpha_{m}^{\mu}, \alpha_{n}^{v}\right]=m \eta^{\mu v} \delta_{0, m+n}, \quad\left[x^{v}, p^{\mu}\right]=i \eta^{\mu v} .}
\end{gathered}
$$


The Hamiltonian of the modes and the operators $L_{m}$ of the Virasoro algebra was usually written in the normal-ordered form:

$$
H=\frac{T}{2} \int_{0}^{2 \pi} d \sigma\left(\dot{X}^{2}+X^{\prime 2}\right)=\frac{1}{2} \sum_{n=-\infty}^{\infty}: \alpha_{-n}^{\mu} \alpha_{n \mu}:, \quad L_{m}=\frac{1}{2} \sum_{n=-\infty}^{\infty}: \alpha_{m-n}^{\mu} \alpha_{n \mu}:,
$$

where $\alpha_{0}^{\mu}=l p^{\mu}$. To find the algebra of operators, we compute the commutator $\left[L_{m}, L_{n}\right]$ by means of the relations:

$$
\begin{gathered}
{[A, B C]=A B C-B A C+B A C-B C A=[A, B] C+B[A, C]} \\
{\left[\alpha_{m}^{i}, L_{n}\right]=\frac{1}{2} \sum_{p=-\infty}^{\infty}\left\{\left[\alpha_{m}^{i}, \alpha_{p}^{j}\right] \alpha_{n-p}^{j}+\alpha_{p}^{j}\left[\alpha_{m}^{i}, \alpha_{n-p}^{j}\right]\right\}=} \\
=\frac{1}{2} \sum_{p=-\infty}^{\infty}\left\{\delta_{m+p} \alpha_{n-p}^{j}+\delta_{m+n-p} \alpha_{p}^{j}\right\} m \delta^{i j}=m \alpha_{n+m}^{i}
\end{gathered}
$$

Rewriting the commutator as:

$$
2\left[L_{m}, L_{n}\right]=\sum_{p=-\infty}^{\infty}\left[: \alpha_{p}^{j} \alpha_{m-p}^{j}:, L_{n}\right]=\sum_{p=-\infty}^{0}\left[\alpha_{p}^{j} \alpha_{m-p}^{j}, L_{n}\right]+\sum_{p=1}^{\infty}\left[\alpha_{m-p}^{j} \alpha_{p}^{j}, L_{n}\right],
$$

and calculating these expressions by using (167), we find the relations for the operator algebra:

$$
\left[L_{m}, L_{n}\right]=(m-n) L_{m+n}+\frac{D}{12} m\left(m^{2}-1\right) \delta_{m+n},
$$

including the anomalous term, or central extension of the Virasoro algebra.

To find out the origin of this anomaly, we calculate the commutator of operators $L_{m}$ in the case when the normal ordering in $L_{m}$ is not performed. Then we have instead of (165):

$$
L_{m}=\frac{1}{2} \sum_{n=-\infty}^{\infty} \alpha_{m-n}^{\mu} \alpha_{n \mu},
$$

and the commutator takes the form:

$$
2\left[L_{m}, L_{n}\right]=-\sum_{p=-\infty}^{\infty}\left[L_{n}, \alpha_{p}^{j} \alpha_{m-p}^{j}\right]=-\sum_{p=-\infty}^{\infty}\left[L_{n}, \alpha_{p}^{j}\right] \alpha_{m-p}^{j}-\sum_{p=-\infty}^{\infty} \alpha_{p}^{j}\left[L_{n}, \alpha_{m-p}^{j}\right] .
$$

By substituting (169) and shifting $p \rightarrow p-n$ the first sum, we obtain:

$$
\begin{gathered}
2\left[L_{m}, L_{n}\right]=\sum_{p=-\infty}^{\infty} p \alpha_{n+p}^{j} \alpha_{m-p}^{j}+\sum_{p=-\infty}^{\infty}(m-p) \alpha_{p}^{j} \alpha_{m+n-p}^{j}= \\
=\sum_{p=-\infty}^{\infty}(p-n) \alpha_{p}^{j} \alpha_{m+n-p}^{j}+\sum_{p=-\infty}^{\infty}(m-p) \alpha_{p}^{j} \alpha_{m+n-p}^{j}=(m-n) \sum_{p=-\infty}^{\infty} \alpha_{p}^{j} \alpha_{m+n-p}^{j}, \\
{\left[L_{m}, L_{n}\right]=(m-n) L_{m+n} .}
\end{gathered}
$$

This is an expression for the Virasoro algebra without the anomaly or central extension. Thus, the source of the anomaly in the Virasoro algebra (169) is the normal ordering in (165). 
Let's consider one heuristic way of obtaining critical dimension for a bosonic string $D=26$. Since $L_{0}$ from (165) there is the Hamiltonian of the string (165), then in the equation for the mass of the physical state $|\varphi\rangle$ :

$$
\left(L_{0}-a\right)|\varphi\rangle=0
$$

the normal ordering constant $a$ is the zero-point energy of the modes. For the appearance of massless states, it is necessary that $a=1$. At the same time, at quantization in the light cone gauge, when only the $(D-2)$ transverse coordinates of the string are quantized, the energy operator of these modes was written as:

$$
\frac{1}{2} \sum_{i=1}^{D-2} \sum_{n=-\infty}^{\infty} \alpha_{-n}^{i} \alpha_{n}^{i}=\frac{1}{2} \sum_{i=1}^{D-2} \sum_{n=-\infty}^{\infty}: \alpha_{-n}^{i} \alpha_{n}^{i}:+\frac{D-2}{2} \sum_{n=1}^{\infty} n .
$$

After regularization, i.e. equating the sum of natural numbers to the Riemann zeta function $\zeta(s)$ at $s=-1$, i.e. $\zeta(-1)=-1 / 12$, were obtained $-(D-2) / 24$ for the last term in (175). Equating this to $-a=-1$ in (174) then gave the critical dimension $D=26$ . Thus, in this method of obtaining the critical dimension, the existence of such a distinguished dimension follows from the existence of zero mode energy.

A rigorous method of calculation of the critical dimension is to find the commutators of the Lorentz group generators $J^{\mu \nu}$. In the calibration of the light cone for Lorentz invariance, the commutator $\left[J^{i-}, J^{j-}\right]$ must vanish, but it turns out to be proportional to:

$$
\left[J^{i-}, J^{j-}\right] \sim \sum_{n=-\infty}^{\infty} \Delta_{m}\left(\alpha_{-m}^{i} \alpha_{m}^{j}-\alpha_{-m}^{j} \alpha_{m}^{i}\right), \quad \Delta_{m}=\frac{26-D}{12}\left(m+m^{-1}\right)+\frac{2}{m}(1-a) .
$$

Thus, the Lorentz invariance condition of the theory $\Delta_{m}=0$, when the algebra is closed, is satisfied only at $D=26$ and $a=1$.

In string theory, it is well known that this anomaly in the Lorentz group is a consequence of the $D$-dependence of the anomaly in the Virasoro algebra (169) at the light cone gauge, where $D$ should be replaced by $D-2$. Thus, the appearance of a critical dimension in string theory is a consequence of the normal ordering of the operators of observables. The conclusions for the fermionic string are similar and therefore the same conclusion is valid for superstrings.

\section{References}

1. Dirac, P.A.M. (1930) Proc. R. Soc., A 126, 360.

2. Pauli, W., Weisskopf, V. (1934) Helv. Phys. Acta, 7, 709.

3. Pauli W. (1943) Rev. Mod. Phys. 15, 175.

4. Pavšič, M. (1999) Phys. Lett. A254, 119.

5. Stueckelberg, E.C.G. (1941) Helv. Phys. Acta, 14(7) 588.

6. Feynman R. (1949) Phys. Rev., 76, 749.

7. Dyson F. J. (1949) Phys. Rev., 75(3) 486.

8. Green M.B., Schwarz J.H., Witten E. (1987) Superstring Theory, v. 1, C.U.P.

9. Bjorken J.D., Drell S.D. (1964) Relat. Quant Mech; Relat. Quant Fields, McG.-H.

10. Itzykson C., Zuber J.-B. (1980) Quantum Field Theory, v.1-2, McG.-H.

11. Zakir Z. (2020) Quant. and Grav. Phys., 1:002-7128.

12. Zakir Z. (2021) Finite Quantum Field Theory, CTPA, T. 